\title{
Seljeplatat
}

fït Den

\section{conargelisdien déligionsuntertidif}

in sexta.

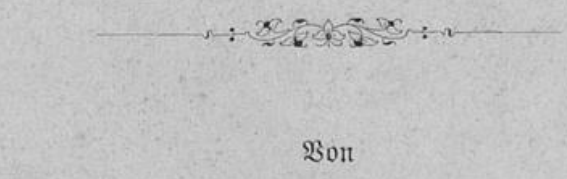

Drof. 12. Tुeititut,

Direkfur Des tiomingl. (6)

1894. \$rogr. $9 i x, 160$.

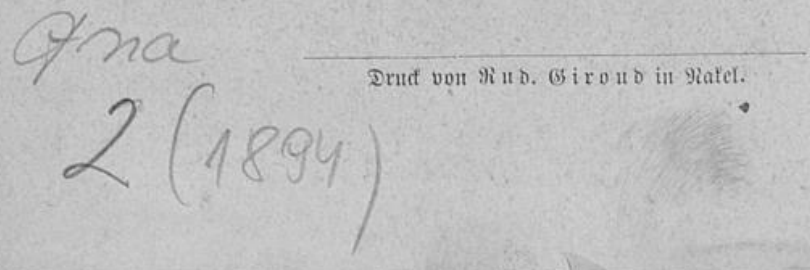




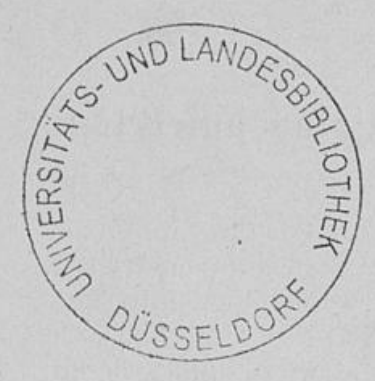




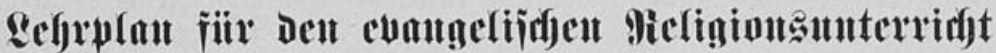 iil Serta.}

SBas id in Der Beilage zum Frogramm Des biejigen fi. Sym=

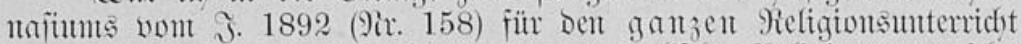
Dargeboten babe, einen Sebrplan für Dent evangelijcben Feligionsunterridst, Das joll mu in biejent gabre uno mit Gottes sülfe in Den folgentoen weiter antsgefülbrt werden für Die einzelnen filaljen, in Der 5̧ofïmung, Damit mtantbent meinter 2Amtsgentoflen, namentlid Dem 2htä̈tger im Sebramt, jeine 2Hfigabe ju erleiditern. Sidbt jeder Religionslebrer ijt Theologe, unto jelbit wemt ex es ijt, jo bereitet ibm Dod ber Unterridst jutächit mebr voer mentiger Edwwierigfeiten. Diejelben fönnten ja viel geringer jein, went für Das Relig̣ionsbud) Des Sdrülers cin finmmentar für Den Sebrer vorbanton wäre; für ein io verbreitetes Budb, wie bas an Der biejigen 2hitalt gebrautbte "Biblijche Sejebuch wou Edbulz=silir", bätte

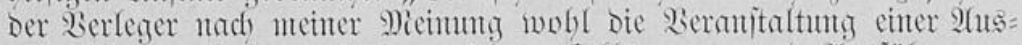

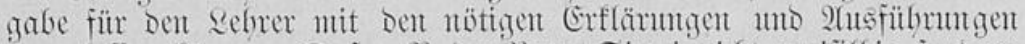

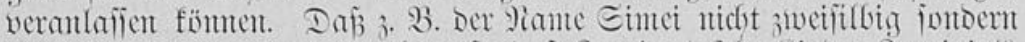

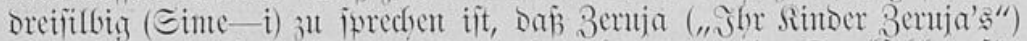

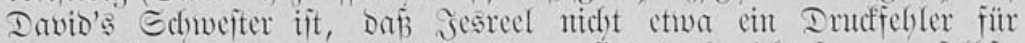

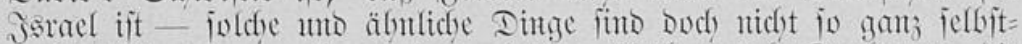
veritänolid, wie mtandber sebrer glaubt. 2Atd) mandse Darlegung, Die

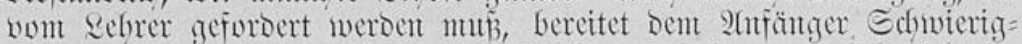

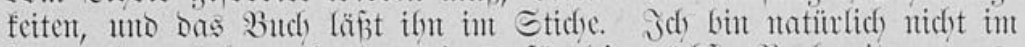
itantbe, Dent seforer ein Derartiges, für bie nächite Sorbereitung auss=

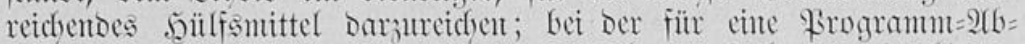

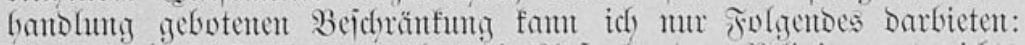

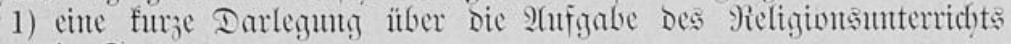
in Serta;

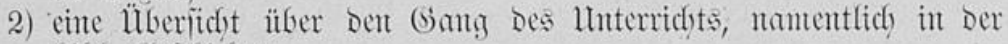
bibl. (Sejchidfte;

3) eintige 2(usfïbnungen jofwierigerer Darlegungen, weldhe Dem Seforer jeine 2 uffgabe etwas erleidtern iollen.

\section{Die Afufgabe Des Religionsunterrid)ts in Serta.}

1. Das Sauptpenium für Den Religionsunterricht in Serta ijt bie Durdunabme und Eimprägung Der biblijchen (Sejdidften Des. Ilten Iejtaments, natürlich nidyt aus ber Bibel, jondern nach einter biblijdsen Sejchidfte, meldye diejelben in einer paljenton 2tuswabl uno einter ant= gemefienen Daritellumig Darbietet.

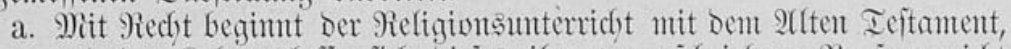
unto ber Sebrer lafje fidh Diejes ifm sorgejdriebente Fenjum nidht verleiden burd bie aud unlängit wieder erbobente Forbenung, Das

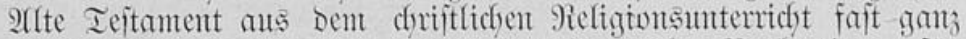
3u bejeitigen. ${ }^{1}$ ) ISent es für Jejum die rechte Sorbereituntg für

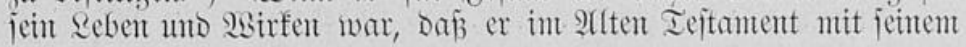

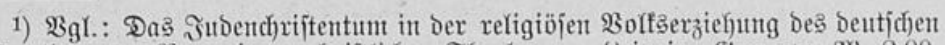

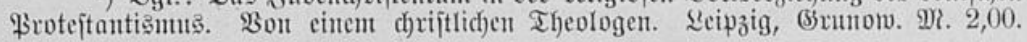


Denfen lebte und mebte, io fant es für ein Efriftenfind wohl auth

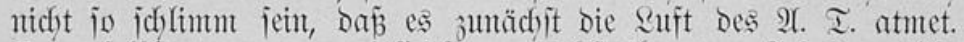
Denn jelbit wenn man bielleidbt zunä d) it ifm von jejus erzäblt,

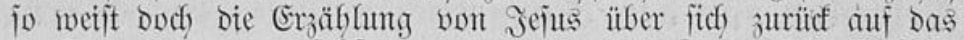
21. T., und es wäre jeltiam, went man Dem Ritnde dieje Riüdfebr jut Sorausjebung bes Chriftentum verjperren wollte.

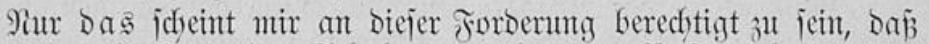
man bas 2 . $\mathcal{I}$. in ber Edfule gegentüber Dem $\Re$. $\mathcal{I}$. nidft in einter jeinem $\mathfrak{U m} \mathrm{m}$ ange entiprechenden 28 eije zur Geltung fommen lafie; bas geichiebt $j a$ aber audh nicht, $d a$ bem $\Re$. $\mathcal{I}$. ebenio viel, bej. ipäter mebr Beit augetviejen wird, als bem 2 . T. Unto auch it Der Gerta - wie bei allem Unterridt im 2 . $\mathfrak{I}$. - mú ber Sebrer immer wieder des Schülers 3 lict auf das $\Re$. . Linlenten; went vom $\mathfrak{A}$ Ilichen Gottesoienit gejprodsen wiro, io mag ber Sebrer aud alsbald vom Nilidben Gottesbienit iprechen; went bie Sejdyidbte vom Sündenfall erzäblt wirb, Dann wirb eine Sin= weijung auf den Erlijier fich won jelbit nabelegen. Sornebmlich

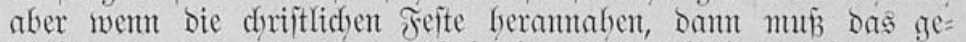
idseben - was ja aud ber Sebrolan ausbrüdfict vorjdoreibt -

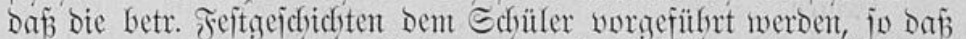
audf in Der Serta das Ebriftentum, die Erfüllung Der 2 Ilichen

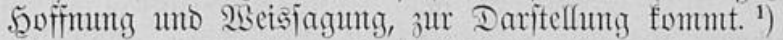

b. 2Benn mun Der Rebrer Die Gejchidste Dem Gertanter erzäblt, Dann

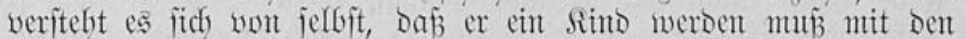
Sinbern. Miag er als Siftorifer mandbe (Sejchidfte anders amieben, als der Sertaner - bier gilt es, alle jeinte gelefurten Gedanten 3urüd überliefenung. Into das ijt fein sacrificio dell' intelletto! Sinter

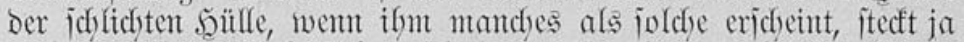
Dod) aud für Den fritijd) gejdulten Sijtorifer ein beiliger fiern:

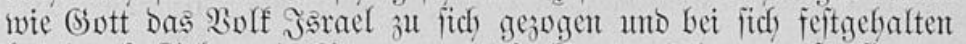
Gat burch Siebe uno Strenge - wie fömte es Dem emitgefintent Foricher, went er jeine Echüler liebt, jofwer fallen, mit Simbern in ibrer Swradje zut reden; alle Fragen uno wollentos alle 3 weifel

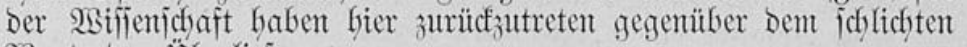
IBorte Der überlieferung.

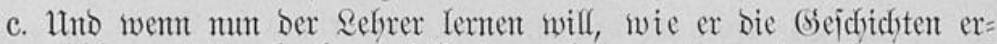
zäblen und Durdfnefomen joll, Dann fant er bas etwa lernen aus einem Budfe wie Edhumann und Eperber, Sejchidste Der Methodif Des Religionsunterridhts in Der evang. Solfsichule (Gotba, Ifiene= manm, 1890, 9X. 2,00), weldbes ifn zugleid) über Den Unterricht im Siatechismus und über die Bebandung des Sirchenliedes orientiert. 2utch findet er mandbe Belebrung über Diejen Interricht in Der Beitidurift für Den evang. Religionsunterridbt (vgl. 3. B. BD. IV, ङ. 195-198: Die "Jormalitufen" im bibl. (Sejchidbtsunterridbt). 2rber jelbit menn Der Sebrer ber metbodijchen Schulung entbebrt,

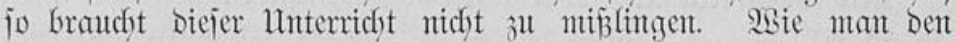

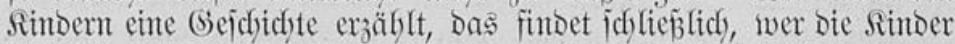

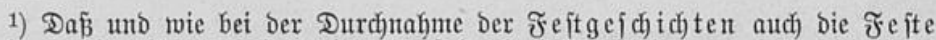
bejprodjen werden fömten, zeigen Die unten folgenten $\mathfrak{A}$ u 
lieb bat, auds won jelber; ja, er wird vielleidft won jelber auf bie jogen. "Jormalitufen" (wentigitents in Der Sauptiache) fommen. Dent einte furze Einleitung wird er oft won jelbit als nötig er=

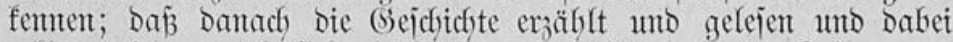
erflärt wiro, ergiebt jich) gleidffalls von jelbjt; Daß̉ eine 3ujammen=

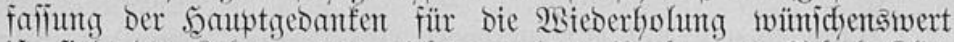

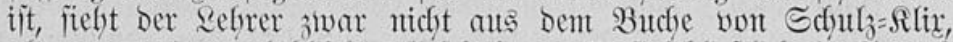

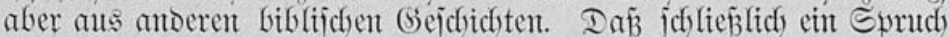

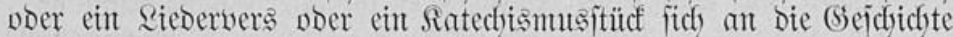
anjobliejen lajie, wird er vielleidbt ebenfalle jelber finton.

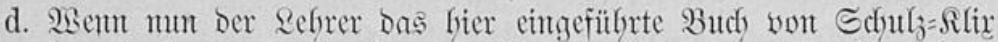
benubt, io mag er beachten, was in Dem \$rogramm bon Burtebube (1887, भir. 313: \$anif), Eintige Bemerfungen über Bibelleftüre unto Sebrbücter für bibl. (Sejchidbte) autd) über biejes Butd gejagt ift. SBie Der Sauptinbalt ber einzelnen (Sejchidsten furz zujammen= gefabit werden famt, fam er lenten bon einem anerfanten Meijter Der

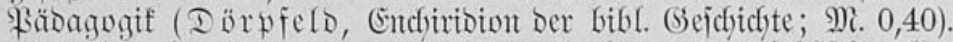

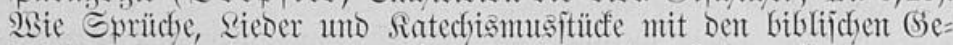
jdjidften werbumben werben fömten, fant Der Sebrer lemten von 3ange, Reitfaden für Den Evang. Religionsunterricht (Seft I, Serta; M. 0,60) uno aus der unten folgenden 2(usfübrung. Went in Der Sdbrift won 3 ange aud Darauf bingenviejen wirb, wie ver=

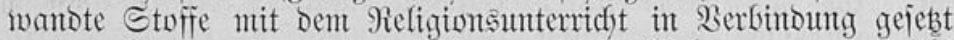
werben fümten, is wird ber Sefrer bieje Mabmung ziwar nicht unbeadjet laffen; aber bier gilt es bejonders, Má̉ zu balten,

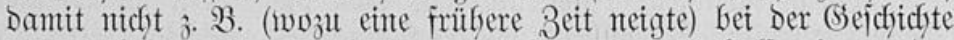
von Jafob's Iod eine lange Daritellung Des Einbaljamierens ber 2̈̈gypter gegeben, Doer beim Durchzug Durch Das Fote Meer eine ausfübrliche Erfläruntg von Ebbe und Flut gegeben werde. Die

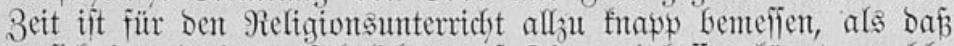
er fich irgendwie ausfïlbrlicher auf Dinge einlajien fönnte, iweldye für bie Feligion bod mur jefr menig in Betradft fommen.

So wird mut Der Sebrer Die biblifdue Gejebidste, weldbe er in Der angegebenen $1 B e i j e$ Dem Sdyüler Dargeboten Gat, für bie nädyjte Stumbe zur 2Biederbolung aufgebent ${ }^{1}$ - aber mur zum erzäblen, nicht зum 2 usmendiglernen. 24 Ento jedes Biertel= jabres (vgl. unten bie überficht!) folgt Dann, nadbem ein befimmter

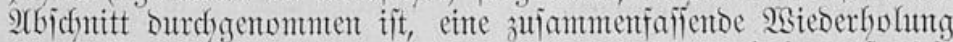

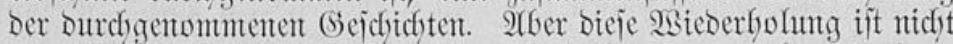
ju einter bäuslidben Âfgabe des S d) ̈̈lers zu madjen, fondern

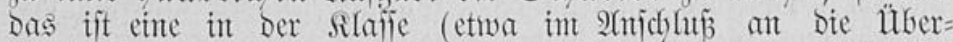
idfriften) worzunefmende 2fufgabe Des Sebrers. Der Sebrer aber wiro fidf) in Der Serta Damit begnïgen, immer ein Bierteljabr jut überblicfen; Das banje etwa immer nochmals zu überblicfen -

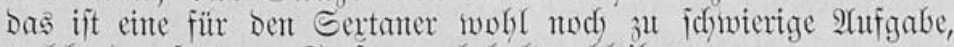
weldbe ben päteren Stufen vorbebalten bleiben mag.

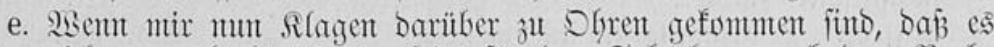
nidft gant leidft jei, einterjeits für Den Schüller, nach Dem Budfe

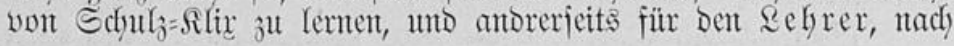

1) Bei einzelnen (sejdjidjten wiro auf biejelbe verzidatet. 


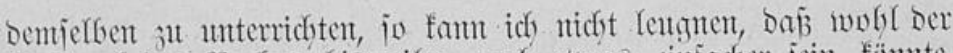
2usbrud bes Buctes bisweilen nud etwas einfacber jein fömte,

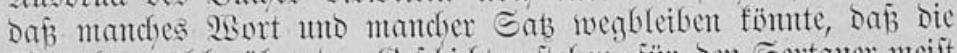
Esrühbe, weldbe über Den Geidsidhten fteben, fütr Den Eertanter meijt

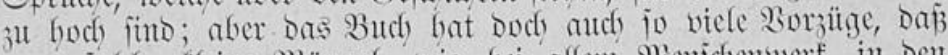
man joldbe fleinte 9)iängel, wie bei allem Mienjchenwert, in ben Siauf nebmen mutb. Der Sefrer aber fundet allerdings, wem er Diejes Budf in bie Sand befonmt, in Den 75 (bes. 77) Sejchidsten,

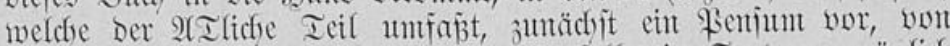
weldiem ex fich jofort jagt, dap̉ er basjelbe in Eerta ummioglich

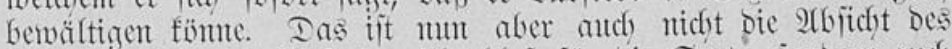
Budbes; biejer Stoff ift ja nidjt bloj für bie Eerta, jondern aud für bie Duarta berechnet, und es gilt aljo, das autsjujcheiden, was für bie Serta erforderlich ift.

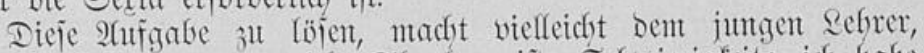
namentlidf went er nidbt Theologe ift, Edymierigfeit; id babe Darum in Der unten folgenden überïtht ( $i x$. II) Dent für Gerta geeigneten Stoif angegeben.

2. Der sweite Segenjtand Des Religiontsunterridyts in Serta ijt Der Ratedismus, won weldsem Das erite Şauptitüct mit Erflänung

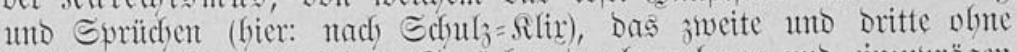

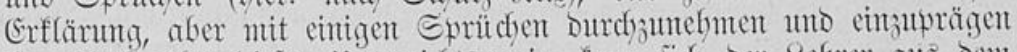
ijt. 2(udb über biejen Itnterridbtszmeig fant fidh Der Sefrer aus Dem oben genamten Budbe von $ఠ$ d)umann umo Sperber vrientieren, wo er auth) auf geeignete bülfsmittel bingemiejen wirb.

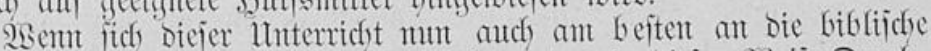

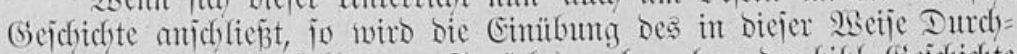

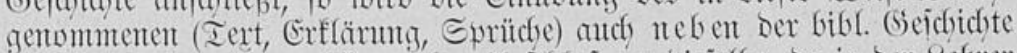

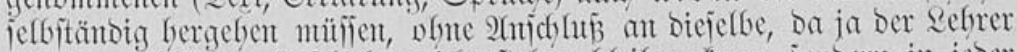
mit ber biblijden Sejofidste nidft iteben bleiben fam, jontoen in jeber Stumbe weitergebt. Daraus entitebt aber aud feine weitere Störung; bie Sieder müfien ja ebenjo, went fie audb juntädjit an bie biblijche Sejchichte angefnüpft werben, Dod) neben berjelben bergebend gelent und wiederbolt werden. Die Theorie, Daj jede Innterridisitumbe ein

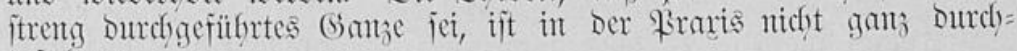
Зutüibren.

A(ud) für Den Interridft int Ratecfismuts gilt es, nantentlich in Den beiden unteren filafien, wo Der fiatedfismus nod) nidbt bie Fiolle ipielen famt, bie er in Dent mittleren filajien ipielen mub: Se einfadser,

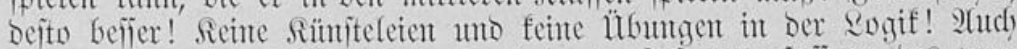
bie am Ende jedes Bierteljabres exfolgente Bujammenfafintg Des son

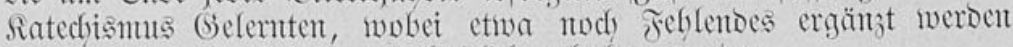
fant, mús recht einfach und findich gebalten werden.

3. $28 \mathrm{ent}$ mun enolich in Serta nod) einige Sieder Durdfgentontmen und eingeprägt werben jollen, io gilt aud, für Diejen 3weig bes Unter=

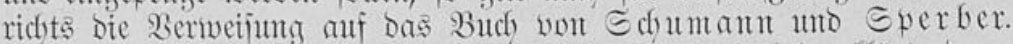
Bunächit wiro natürlict) Das Sied vorgelejen und Dabei erflärt, Dam

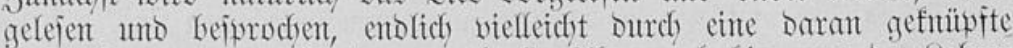

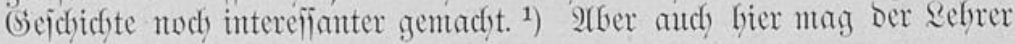

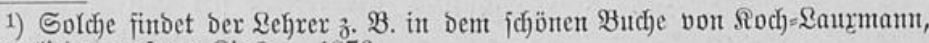
Die Rerntieder unjerer Rirdje. 1876. 


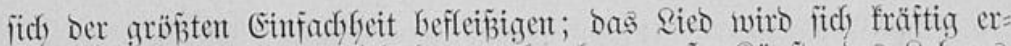
weijen an Serzen Des Échülers aud) obne grof̉e fiülte Des Sebrers.

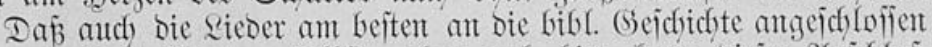

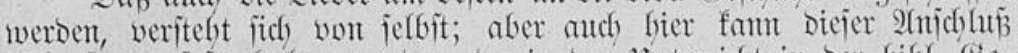
nicht itrentg feitgebalten werben, oa ja ber Interridbt in Der bibl. (Se=

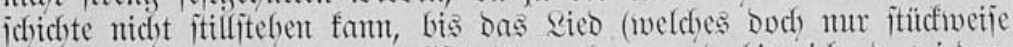
aufgegeben werben famt) vollftündig gelemt und bintreichent wieder= holt iit.

4. פgeru mut int Sebrwlan gefordert wird, Daß̧ "alle Teile biejes Unterriduts (bibl. Sejchichte, fiatedismus und Sied) in lebentoige $B e=$ jiefung [3u-eintander] gejebt merben", is ift bieje Forberung natünlicf) Durdbaus beredhtigt; aber es wiro Dent sebrer nidft Durdweg gelingen, ifor geredst ju werden. Miag aber mun ber sebrer audf mux jeden einjelnen Segenitand in angemeflenter SBeije bebandeln, is miro ber

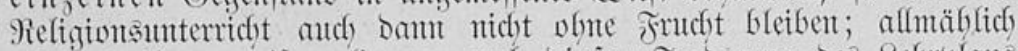
wiro es ibm genip gelingen, aud biejer forberung Des Sebrolans immer mebr zu genügen; wie biejer fovberuntg bei einzefnen Gegen= ftänden Des Anterridbts genïgt werden faum, zeigen bie unten folgenden 24ıรfübrumgen 9 ix. 3,4 uno 5.

5. SEent mun Der Sebrer überblicft, was er in Serta am Ende

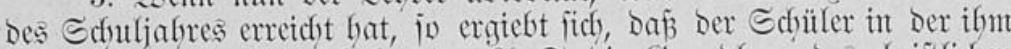

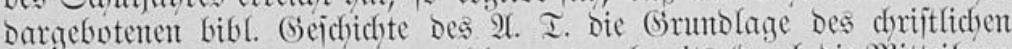
Slaubens gemonnen bat, von weldber aus er bereits (burd) Die Mitteilung

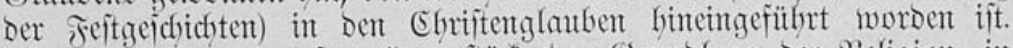
Ebentio it er vom eriten Sauptitüd, Der Srumblage Der Feligion, in bas Eentrum Derjelben, Den SSlauben und das (Sebet, fineingefülbrt worben. Demielben 3wecfe dienen audh bie won ifm gelernten Sieber. In jeinen Elnriftenglauben ifn genauer und vollitänoiger einzu= fübren - Das ijt die 2lufgabe der Duinta.

\section{II. itberifdit.}

\section{Erites Bierteljabr. $\left.{ }^{1}\right)$}

A. Wie bie Menichen won Gott gejdraffen worden, aber won ifym abgefallen finto.

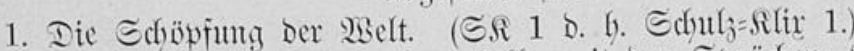

Siat. II, 1 (Iert, obne Erfl., mit Den Epriädent: \$5. 90, 2.

104, 24. 106, 1.) Siat. I, 1. (İert mit Erfl. unD Den Eprüd)ent:

Mit. $10,28.1$. Jof. 4, 19. \$i. 3\%, 5.)

Qieb: "D Gott Dit frommer (Sott."

2. Das Barabies uno ber Sümbenfall. (ङR 2.)

Sirad) 21, 2. Rat. III, 5. 6. 7 (mur Iert).

"D Gott Dut frommer Gott" (Bers 1).

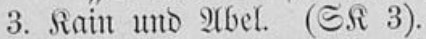

Siat. I, 5 mit Exfl. uno Evrïdben.

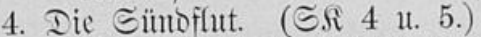

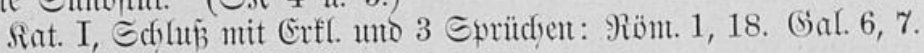

Эei. $54,10$.

1) Rieber: D Sott Du frommter Bott.

Wer mur ben lieben Bsott.

Rated)i 
5. Toab's Radjfonmen; Der Tumbaut ju Babel; Das Sceidentum ${ }^{1}$ ). (ङS 6 u. \%).

fiat. I, 1 mit Erfl. und Sprïcben, nebit Biloerverbot.

B. 2Bie Gott Die Stammbäter Des 2olfes Sorael im redstent

(S)lauben erbalten bat.

6. 2tbrabant's Berufung; Rot's Iremunty won 2tbrabant; Sot's Ev= rettumg Durch) 2ffrabam. (Sศi 8 u. 9.)

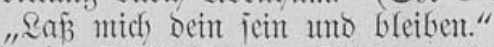

\%. 2Abrabam's Fürbitte für Sobom uno (Somorra; Der Itntergang oer beiben Stäbte; Sot's Errettunty. (S.i 11 u. 12.) ${ }^{2}$ )

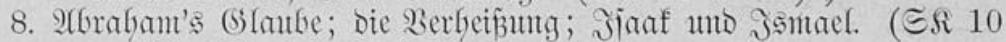
u. 13.) Smrüdbe Sal. $10,1$.

9. 2tbrabam's Brüfung. (S. 14.)

Siat. I, 1 mit Ertfl. unt 3 Esrüctsen: Mit. 10, 28. 1 job. 4, 19. Pुi. $3 \%, 5$.

10. Jiaaf und Rebeffa. ( $\subseteq \Re 15.)^{3}$ )

"2Bלer mur Den lieben Gott läß̈t walten."

11. Sarab's und Albrabam's IDd und Begräbnis. ${ }^{4}$ )

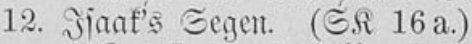

fiat. I, 2 mit Erffl. unto 3 Sprüdben: Nit. 12, 36. Seebr. $6,16$. Mit. 5,37 .

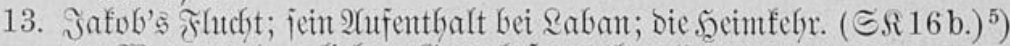
"2Ber mur Den lieben Gott läß̈t walten."

14. Jafob's und Elau's Seriöbmung; Jianf's Iod; Die Yadbfommen Gjau's. $\left.(\text { Sfi 17. })^{6}\right)$

1. Moie $32,11 \mathrm{a}$.

15. Sojesf und jeine Brüber. (ธЯ 18.)

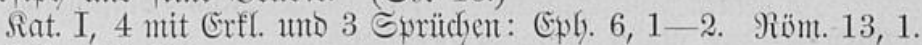
5ebr. $13,1 \%$.

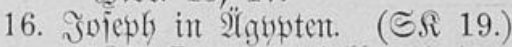

Rat. I, 6 mit Erfl. und Dem Eprudbe 1. Noje 39, 9.

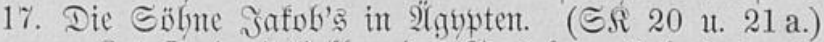

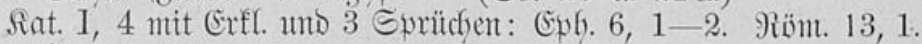
Sebr. $13,1 \%$.

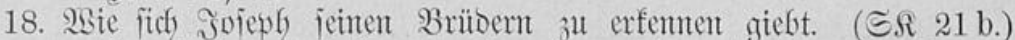
Siat. I, 8 mit Erfl. uno Enutb: "Şbr gedadbtet es bije mit mir zu madben, aber (Sott gedadbte es gut zu madben."

19. Jafob und bie ङeinten in 2̈̈gipten. (ङS 22.) ${ }^{7}$ )

"SBer mux Den lieben Gistt läß̈t waltent."

3ufammenfaijung und 2Biebergolung.

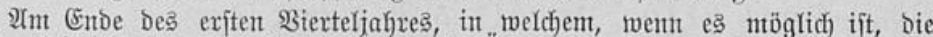

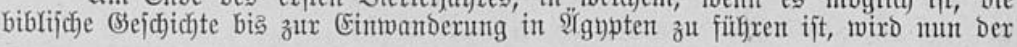

1) Som Seibentum ntag Dex Sefyrer fiter ipredjen nad) 2(poítelgejd). 17, 26-28 uั Röm. 1, 21-23.

2) Nux zu Yejen oder zu erzählen, nidjt zu wieberbolen!

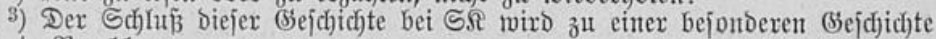
erweitert: গx. 11.

4) J゙ehlt bei SR; nux exzählen, nidjt mieberholen!

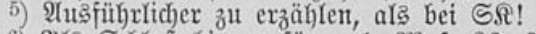

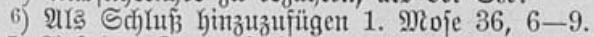

7) 2uf Den Segen Jafob's ntag Der \&ebrex in Serta verzidyten, aber von

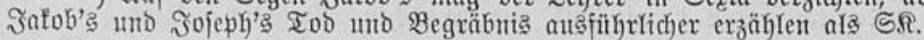




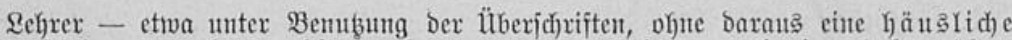
Yufgabe zul madjen - Die Durd)gentommenen (Sej(bid)ten nod) einmal furz über: blicfen und zujanmenfafjen (1 Stunde).

Ebenjo mögen bie gelernten (Sebote und Sprïche nod)mals wieberfyolt werden;

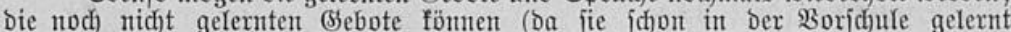
worben find) bei diejer (Selegenheit (mit Erth., aber ohne Sprïd)e) ebenfalla wieber holt werben. Audh aus biejer \$stederbolutg ijt aber für bie Schüler nidgt eine

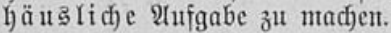

$$
3 \text { weites } \text { Bierteliabr. }{ }^{1} \text { ) }
$$

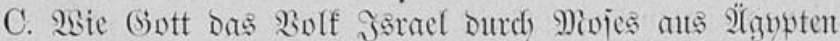
erlojit und mit bem Bolfe einen Bund gejdslofien bat.

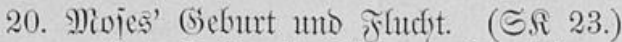

fiat. I, 5 mit Erft. mo Surüctren.

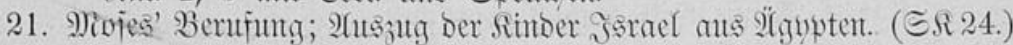

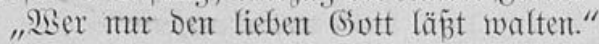

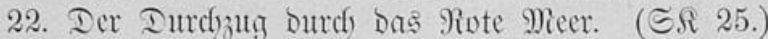
"Sobe Den Serren, Den m. Si. Der Efrren."

23. Der 3ug zum Sinai; Die Gejebgebuntg. (Sfi 26 u. 27.)

3. Moje 26, 12. Sich will euter (Sott jein, uno ifr jollt mein \$olf jeint.

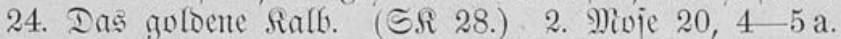

25. Der 2ufenthalt an Einai. (ङS 29.) Sat. I, 3 mit Ertl. ${ }^{2}$ )

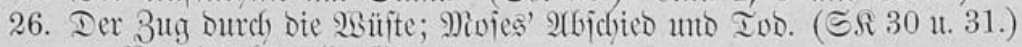
"Tiun Danfet alle Gott."

27. Jojua's Berufung; Die Sundichafter; Der Bug itfer Den Sordan. (ङSi 32a.) "Tiun Danfet alle Gott."

28. Die Eroberung won Jerichn; 2 deban's Diebftabl; Die Rift Der (Gibeoniten. (ङన 32 b u. 33.)

Siat. I, 7 mit Erfl. und 2 Sprüchen: 1. Tim. 6, 6 und 6, 9-10.

29. Jojua's fernere Siege und jein IDD. (Sภi 34.); Das heilige Sant. ${ }^{3}$ ) "Niun Danfet alle Gott."

Bujammenfaijung und 2Bieberbolung Der Dutrdgentumenten bibl. Sejchidbten und won Siat. I, wie am Enbe Des worigen Sierteljabre.

\section{Drittes Bierteljabr. $\left.{ }^{4}\right)$}

D. 2Bie Gott im Solfe Jarael bas fiönigtum aufgerichtet und Durch Die Fropheten ju jement Bolfe geredet bat.

30. (Sidenn. (ES 35 น. 36.)

Siat. I, 1 mit Erfl. ıno 3 Spritdent: Mit. 10, 28.1 Job. 4, 19. \$3i. $37,5$.

1) Sieder: Sobe Den 5erten.

Pun Danfet alle Brott.

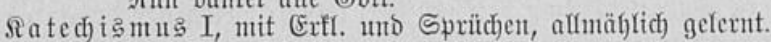

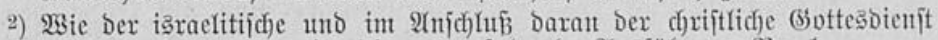

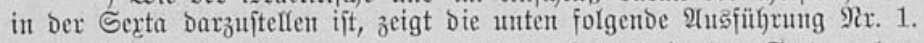

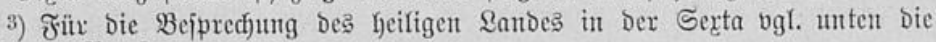

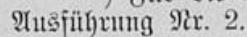

4) Lieber: Mit Exnit, ihr Menjobentinder.

Dies ifft Der Tag.

Ratedismus I, mil Erfl. mo Sprüdjen. 
31. Jephtha. (GSi 3\%)

Siat. I, 2 mit Erfl. un 3 Enrïdsen: Mit. 12, 36. Mit. 5, 37. \$5i. $50,15$.

32. Simion. (ङన 38.)

fiat. I, 2 mit Erfl. uno 3 Sprüd)en.

33. Rutb. (ङЯ 39.)

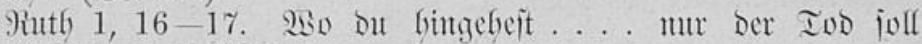
mich) unto bidf icheiden.

34. Eli uto Samuel. (ङ. 40.)

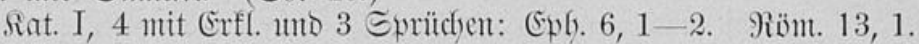
Sebr. 13,17 .

35. Saul wiro Rïnig über Э̧srael. (SSi 41.)

Siat. I, 4 mit Erfl. umo 3 Eprïtsen.

36. Saul's Seldentbaten und jeine Berwerfunty. (ङ.R 42.)

1. Sam. 15, 22. Geborjam ift befier... 2Bidoern.

3\%. Davio's erite Salbung. (S.R 43.)

"Ein Mienich fiebet, was vor 2tugen ift; Der Serr aber fiebet Das ber ant."

38. Davio uno Der Fieje Goliatb. (Sfi 44.)

Bisialm 124, 8.

39. David und Jonathan. (ES 45.).

Siat. I, 8 mit Erfl. unt Evmud) Eph. 4, 25.

40. Sant und Tavid in Der 5öble. (ङS 46.)

9) it. $5,44-45$.

41. Saul's Berzheiflung uno Enoc. (ङЛ 47.)

fiat. I, 2 mit Ertl. uno 3 Emrüdsen: Mit. 12, 36. Mit. 5, $3 \%$. \&i. $50,15$.

42. Davio als fionig. (Sోi $48-50$.)

Midua $5,1$.

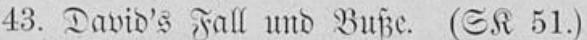

"Ein reintes Secr, Serr, ichaff' in mir" (B. 1).

44. 2tbjalom's Empörumg. (S. 52a.)

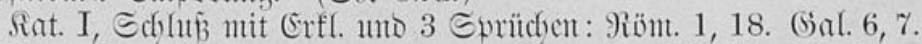

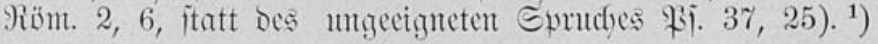

45. 2tbjalom's Ende. (ङS 52 b.)

fiat. I, Edbhí mit Exfl. uno 3 Evrüiden.

46. David's lebte Tage; Der Sibnig Salomo; die Immelneifue. (ङS $53-55$.)

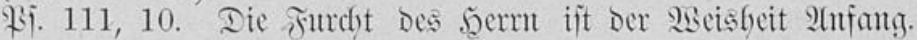

47. Ealomo's serrlichfeit uno Fall. (ङన 56.)

Siat. I, 1 mit Ertl. und Evrudf Miatth. 4, 10.

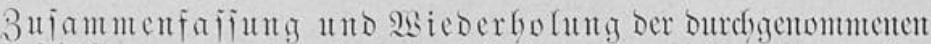

bibl. Gejchidften und Des eriten Sauptitiofs, wie ant Ende Des erjent Bierteljabres. Darauf folgt (9 Et. - wor SEeibuaduten) Die $\mathfrak{B e}=$

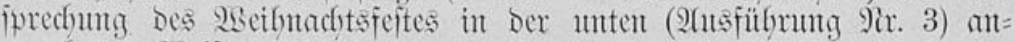
gegebenten 2 seije.

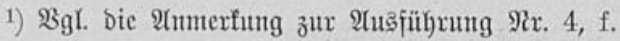




\section{Biertes Bierteliafr. ${ }^{1}$ )}

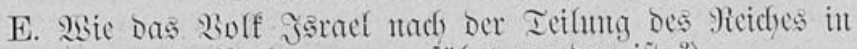
die Berbanmung gefiifont mordent iit. ${ }^{2}$ )

48. Rebabeam und Jerobeam. (ङ.R 5\%)

2. Mivie 20, 4 5 a.

49. Der \$rophet Elia. (Si 58.)

fiat. I, 1 mit Erfl. uno Spruth Dit. 4, 10.

50. 2hbab und Siebel; Der Frophet Ëlija. (ङS 59 u. 60.)

fiat. I, 8 mit Erfl. uno Entud) Eph. 4, 25.

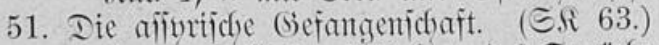

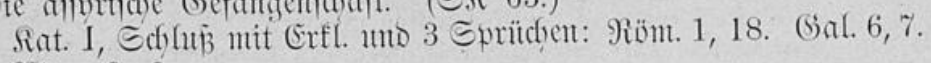
Yïm. $2,6$.

52. Sistia uno Sanberib. (ङS 65.)

fiat. I, 1 mit Erfl. uno 3 Exridden: Mit. 10, 28. 1. Jofs. 4, 19. Pi. $3 \%, 5$.

53. Der Riönig jofita. ( SR 6\%)

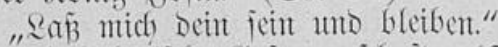

54. Die babuloniphe (Sefangenidraft: (ङS 69.)

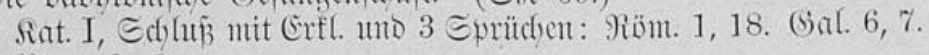
Rïm. 2, 6.

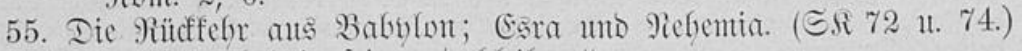

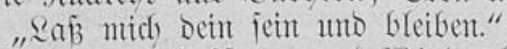

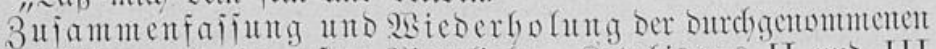
bibl. Siscicfidsten, wie int erjten Siertefjabr. Siatedrismus II unto III fint jebn ant 2 ntiange Des Bierteljabre wiederbolt worden. $2(m$ Sd halie des Bierteljabrs erfolgt, wie jobn wben bentert, Die Be= iprechung Des Diterfertes (2Usfübrumg Yir. 5: 9 Stunden).

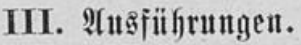

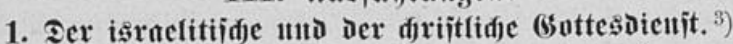

A. Der israelitijue Gottesdientit.

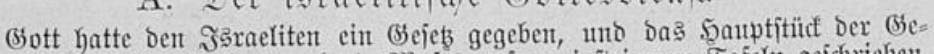

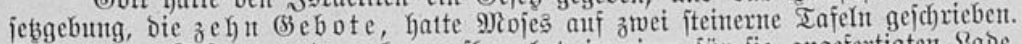
Dieje beiden Tafeln wurben aber aufbetwah) in einer für fie angefertigten \&abe, welde bie $\mathfrak{B}$ un desla de gentant wurbe, weil in ihr bas Sejebs Sottes aufbetwahrt

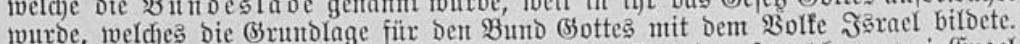
Die Bunbeslabe war aber gej(f)lofien burch) einen Decfel, auf weldjem zwei Engel itandert.

Für bie Bundesłade murbe die Stiftshütte angefertigt, o. hy. eit Belt,

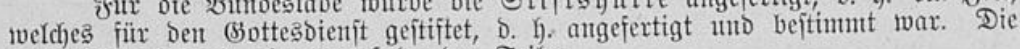
Stiftahnitte beitanto aber aus folgenden Teilen.

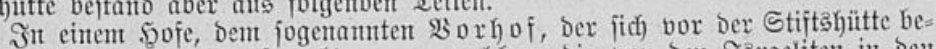
fant, itant Der $B$ randopferaltax, an weldjent bie von ben Jaraeliten in bent

1) Sieber: Gott Deş ફrimmels unto ber Erbent.

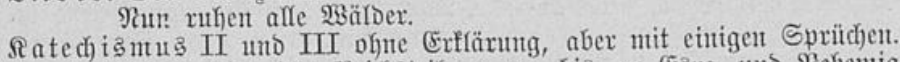

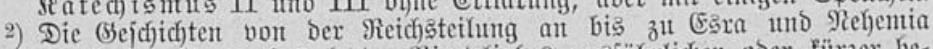
miiffen je nad) ber Dauer Des Yebten Sierteljafrs ausfülyrlidfer ober fürzer bes hanbelt werdent; obent finto mur bie unentbehrlidjen (Sejejidjten angegeben. Dex

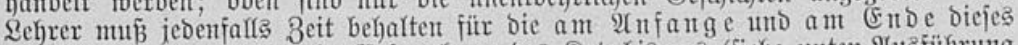

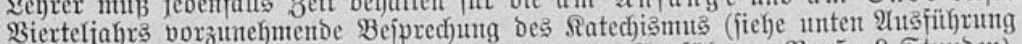

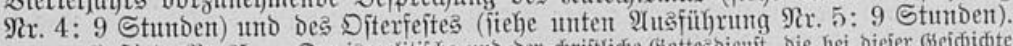

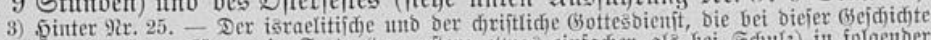
beiprodjen werben mögen, fömen it Serta (ber eritere etwas einfadjer als bei Sd)uls) in folgenter iseife bargejtellt werbent. 
Sorfyof gebradjten Tiere Sott geopfert wurben. Die Stiftähütte felbjt war ein auß 3wei Teilen beftehentes Belt; im vorderen Teile besjelben, Dem Seiligen, itanden

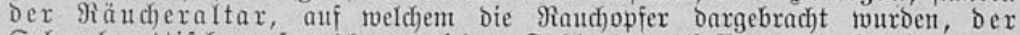
S d)aubrottifd), anf welchen an jebcm Sabbath zwölf Brote als Danf für bas von (Sott gejdenfte tägliche Brot gelegt wurben, unto ber jiebenarmige

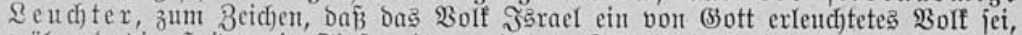

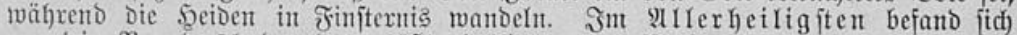

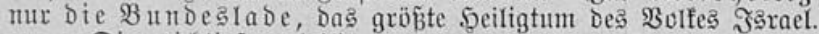

Die midftigiten heiligen Sanolungen ber Jaraelitent waren bie Dpfer. $23 e r$ opfern wollte, brachte ein feflllofes reines Tier zur Stiftæhütte; Der \$riéter ichlachtete Dasjelbe, fing Das̉ Blut in einem Becfent auf und bejprengte Damit Den 2iltar; Darauf wurbe Das Iier ganz voer zum Teil verbrant; Der Dpfernde hoffite

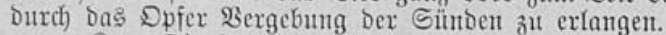

Bum Dienfte am Seiligtum war aber ber Śtam 2 evi beftinmt; ein

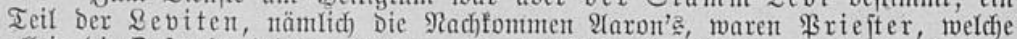
allein bie Dpfer Darbringent Durften. 2In Der Epibe Der 'Brieiter itand Der 5 o he $=$

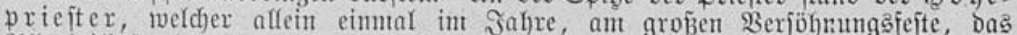
2Allerheiligite betrat, um Den Dectel Der Bunbešlade mit Dpferblut zu beiprengen

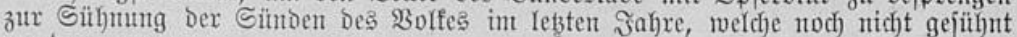
worben waren.

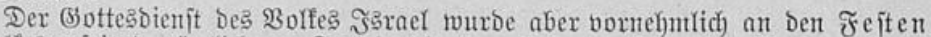
Des 230 lfes gefeiert, nämlich am Sabbath, Dem fiebenten Tage Der $230 d j e$, uno an Den brei gropen Jalieşeften:. Ditern oder Baffahfeit, gefeiert zum Un=

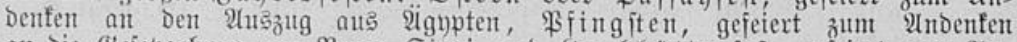
an bie B̈ejebgebung am Berge Sintat, uno $\mathscr{L}$ a Denfen ant Den 2Buitenzug, wo bie J'sraeliten in Şütten, D. h. Belten, gewohnt

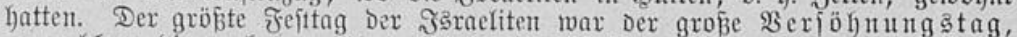

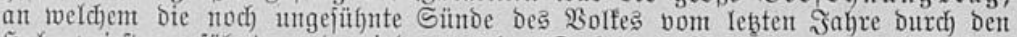

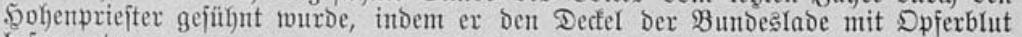
bejprengte.

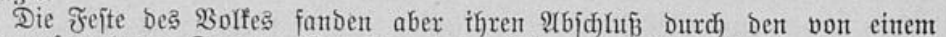

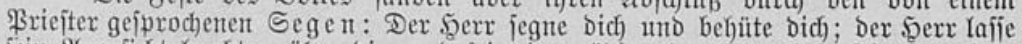
fein 2ngeficht Yeudjten über Dir und jei Dir guäbig; Der şerr hebe jein 2ntgejidjt iiber bich) uno gebe Dir Frieben!

\section{B. Der driftidse Gottesdienit.}

Ein joldbes (5)tteshaus, wie bie jübijdje Stiftăhütte (unto jpäter ber Tempel) war, in weldem Durd Priejter Dpfer gebrad)t werden, haben wir (Ehrijten nicht; wir (E)riften bringen feinte Dpfer mefr, um Sergebung ber Sünden zu er-

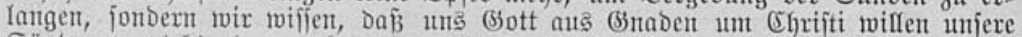
Giindent bergiebt; Darum braudjen twir aud feine Dpfer unt feine \$riejter, und unjer Sottesbienjt iijt nicht, wie bei Den Stuben, an einen einzigen Dort gebuntoen,

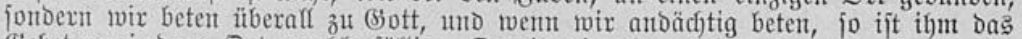
Bebet ant jebem Drte twohlgefällig. Damit wir aber aud) zujammen beten und

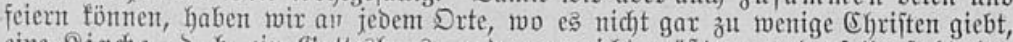

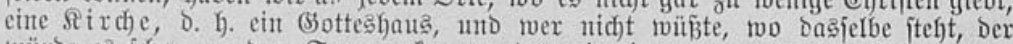

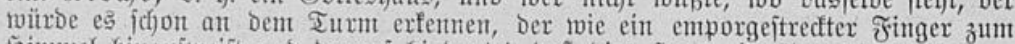

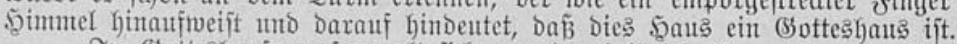

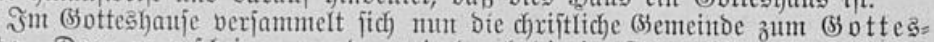
Di enfte. Dału zu exicheinen, werben wir Durd) Die im Turm Gängenden Ǵlocfen eingelaben, und 1venn wir in Die Sirtche eintreten, fo ertönt, weun Das \&äuten Der Biloden aufhört, Die auf einem Ehor angebrachte Drgel, Las getwaltige Jnjtrument,

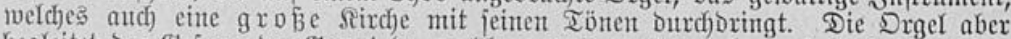
begleitet Dent Bejang Der (3emteinde, welche aus Dem (Bejangbuth) eints ober mełrere

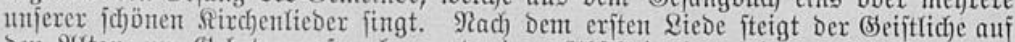

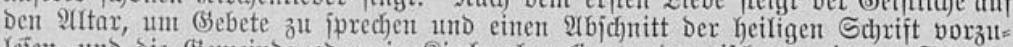

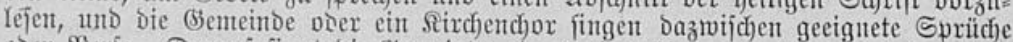
ober Serje. Darauf fingt bie Bemeinde ein ziveites \&ieo, unt alsbant befteigt Der

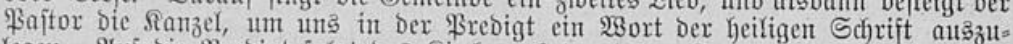
legent. 2tuf bie \$rebigt folgt Daß Rirchengebet unt ber Segent, unt zowar Derjelbe,

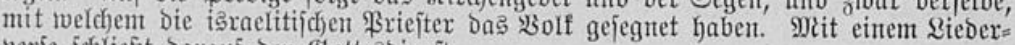

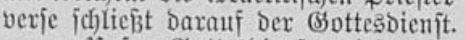

Unjer Bottesbienjt wiro aber vornebutlid an Sonntage uno an ben Jefttagen abgehalten. WSir (Ehriften feiem nicht melyr, wie bie Juben, Den 


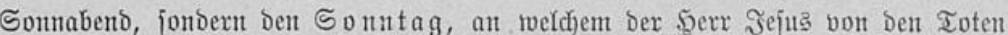
auferitanden ijt. Dagegen feiern wir ebenfalle, wie bie Juttoen, Das $D$ it ex f e it,

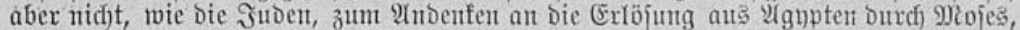

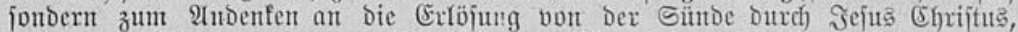
weld)er an Cryarfreitag um unjerer Sinnden willen gef́torben und an Diterjoming

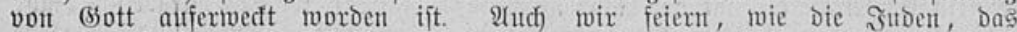

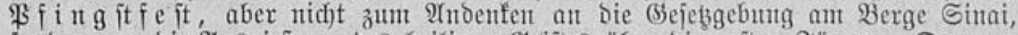

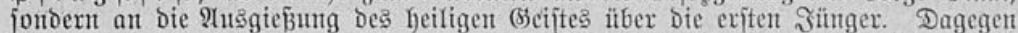

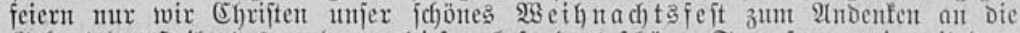
(Beburt bes Seilandes, und von Diefem bejonders jdjönen Iage jagen wir mit bem fromment Didfter (Sellert:

Dies ijt Der Tag, Den (Jott gemad)t;

Sein werd' in aller $25 e l t$ gebad)t!

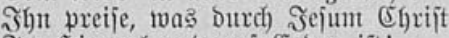

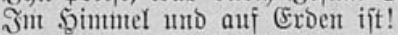

\section{Dns fjeiltge Sano. $\left.{ }^{1}\right)$}

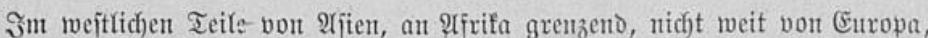

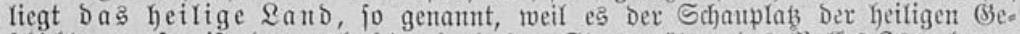

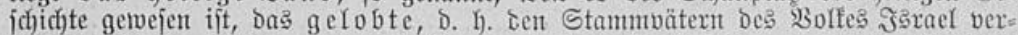
beiBene $\mathfrak{Q} a n \delta$, in ber alten Beit $\mathfrak{R}$ ana an oder $\mathfrak{B}$ a Iäjtina genannt. Dasjelbe

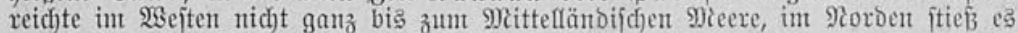

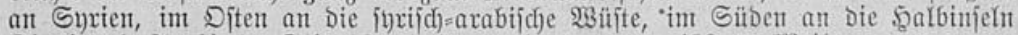
Sinai und थrabien. Seine 2usbehnung betrug etwa $400 \square$ Meilen.

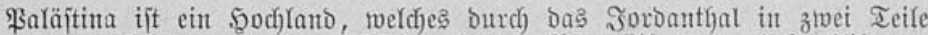

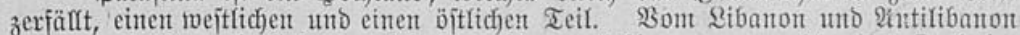
her, bent früber mit Eebern reid) betwachjenten (Sebirgen, an welche bas Santo int

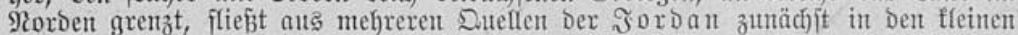

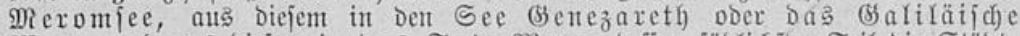
Meer, und aus biejem in bas Tote Meer, Defilen füblichiter Deil bie Stäbte Sodom und SBomorra ïberitrönt hat. Diejes Deer liegt $400 \mathrm{~m}$ unter Dem Spiegel Des 9littelländijđjen Meeres - jo tief unter Den s)ieeze, wie feine andere Stelle ber Eroe; es ijt ein totes Meer, weil es jo viel Salz enthält, bañ fein Tier in jeinemt Wajier leben faun. 2us Diejent Meere fann ber Jorban nicht

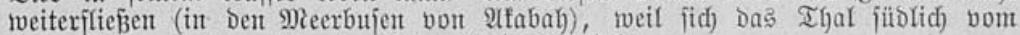
Toten Meere wieber über Den Mieeresjpiegel erhebt.

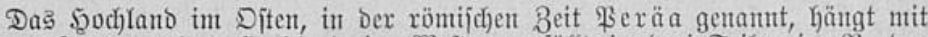

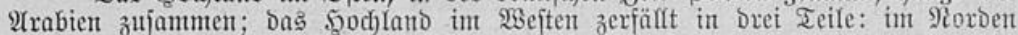

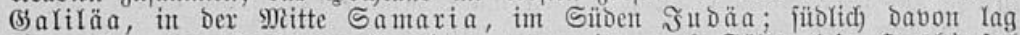

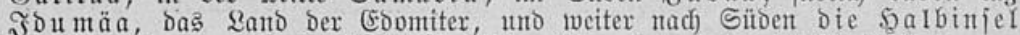

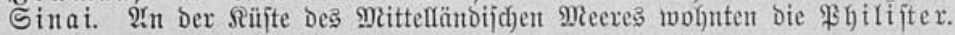

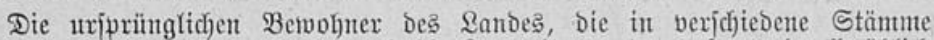

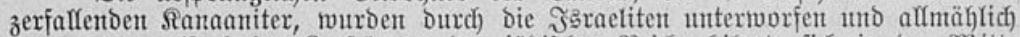

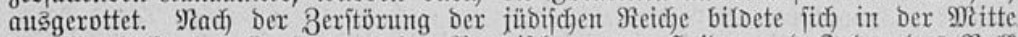

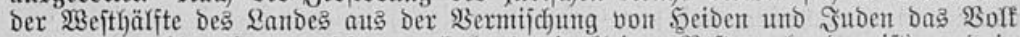

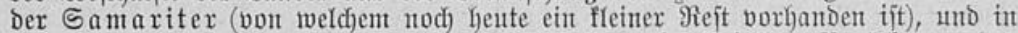

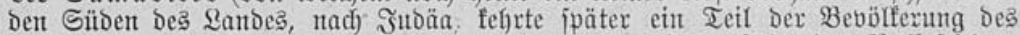

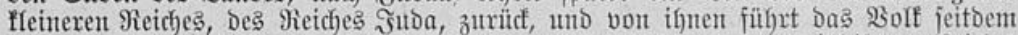

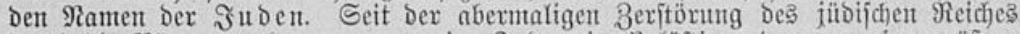

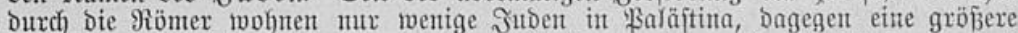

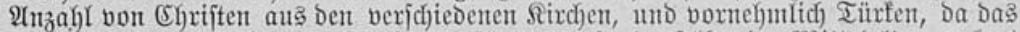
Lanto ja gegenwärtig Den Türfen gehört, weldje basjelbe im Mittelalter erobert und gegen Die von Den Egriṕten unternommenten firenzzïge behauptet haben.

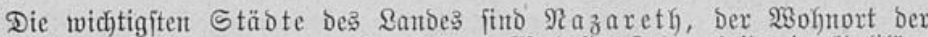

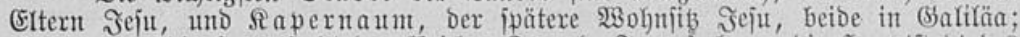

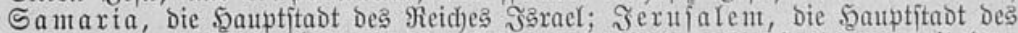

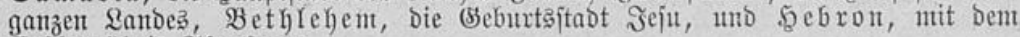

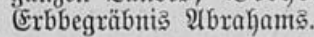

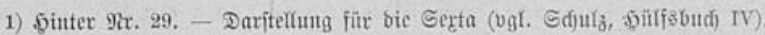




\section{Dิก 23eifund}

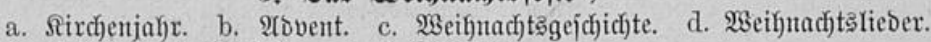

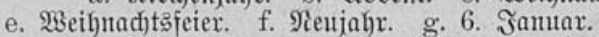

a. Wir haben ant lebten Somntage eit nenes Rird)enjahr angefangen,

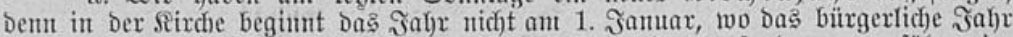
anfängt, ober nad) Dítern, two unjer Sdjuljahr anjängt, jonderı ungefälyr vier

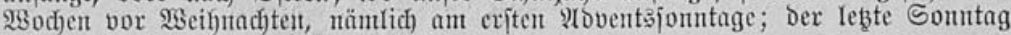

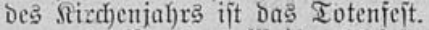

b. Bor Dem Weihnachtsfeite nämlid, feiern wir viex 2 bentsionntage,

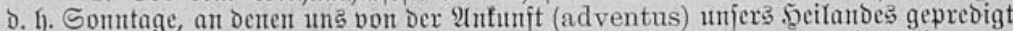

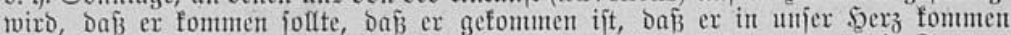

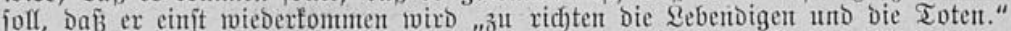

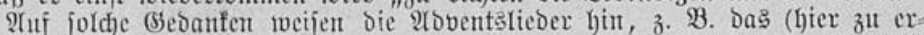

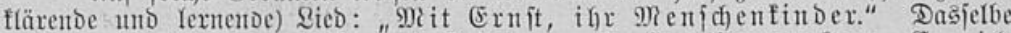

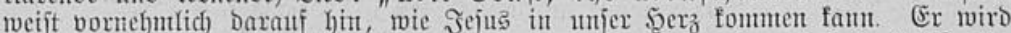

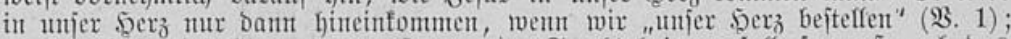

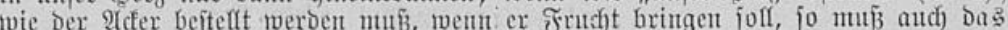
Serz beitellt werben, went Das Rommen Des Seilanbes für uns Frudft bringen

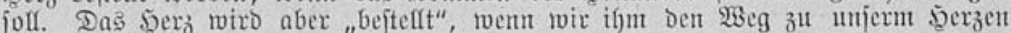
"bereiten" (\$3. 2), went mir" "jeine Steige ridjtig, afle Babnent red)t, Das sirummte gleidh uno idjlecht, ठ. h. gerabe madjen", D. h. ilym einen geraben Weg zu unjernt

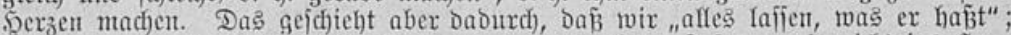

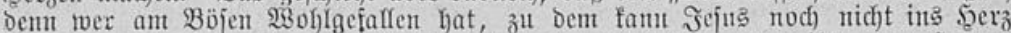

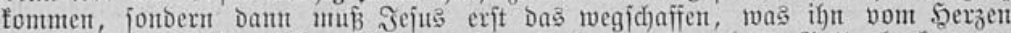
fernhält; Die Igale nüijen erhöht werben, D. h. Iwer ganz von Bsott abgefommen

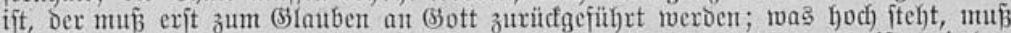
erniebrigt merben, D. h. wer nad) jeiner Meinung id)on fromm genug ift (wie ber

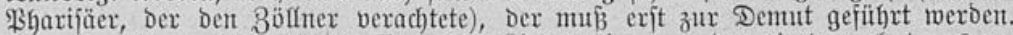

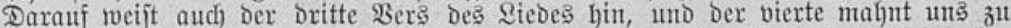

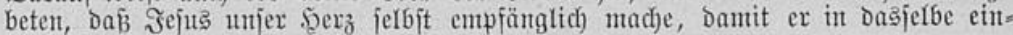
zieken fönne.

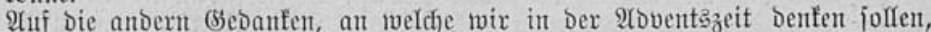
tweijen uns andere Sieber hit, 3. $\mathfrak{B}$. auf jeine Wsieberfunit Str. 10 bes \&iebes:

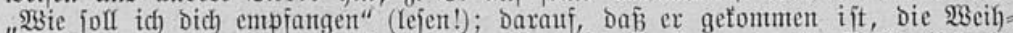
"nad)tslieber, 3. 9 . Str. 1 Des Siebes: "Sott jei Daut Durd) alfe $29 e l t "$ (Iejen!).

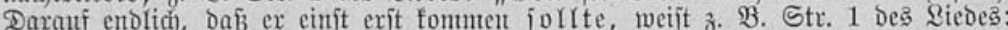

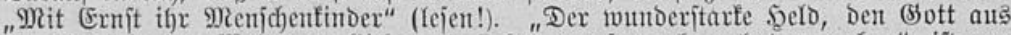
"Snad" allein ber Welt zum Sidjt und Qeben vexiprodjen hat zu geben", ijt vor

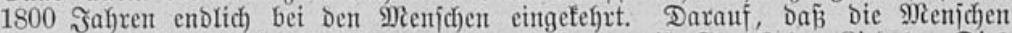

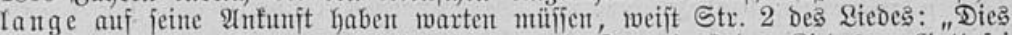
ijt Der Tag, Den (Sott gemad)t" (Iejen!), und Strophe 2 Des Riebes: "Sott jei Dante Durch alle 23 elt" (lejen!).

2 as aber Die $\mathfrak{B a ̈ t e r}$ erwartet und die \$ropheten getweisjagt haben, Das jagen uns Strophe 4 utb 5 Des Qiebes: "Robt (Śott ihr (Ehriften alle gleid)" (leien!).

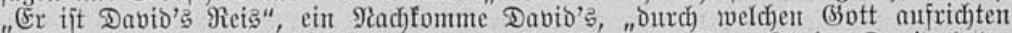
"wollte" Das vollfonmente (Sottešreidh, Die Ehriftenheit. Dem Rönige Davio hatte

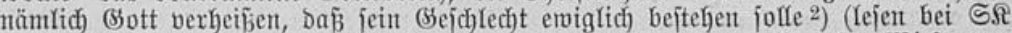

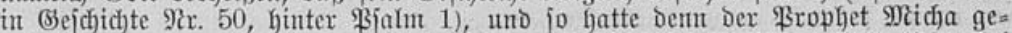

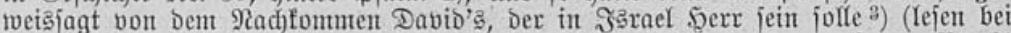

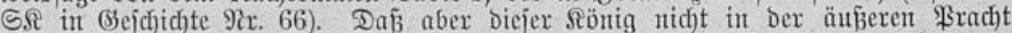

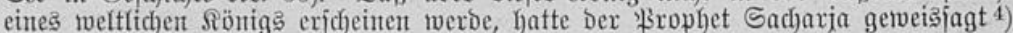
(Iejen bei SR in (Sejd). Nix. 75). 5)

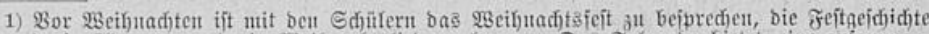

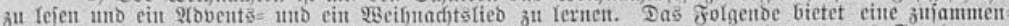

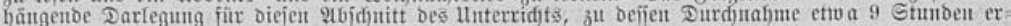
forberfid jeint werben.

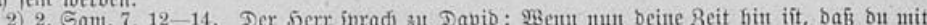

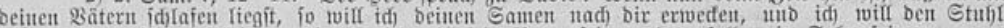

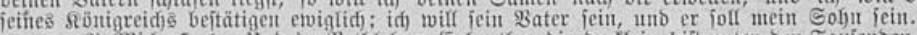

3) Midja 5, 1. Lnd bu Bethlehen (Ephratha, bie buflein bijt unter ben Iaujenben in Juba,

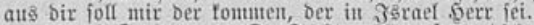

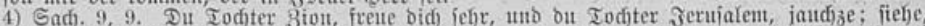

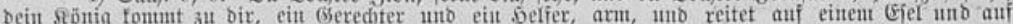
einem jumgen filltent ber 'Elefin.

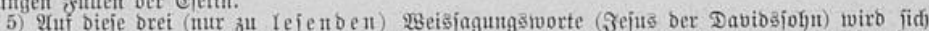

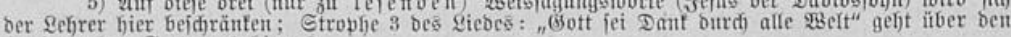
forizont des Sextanters hitaus. 
c. $\mathfrak{W a}$ Der alten $\mathfrak{B a ̈ t e r ~ S d j a r ~}$

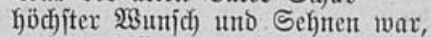

unto was fie gevrophezeit,

ijt exfürrt in Serrlichfeit -

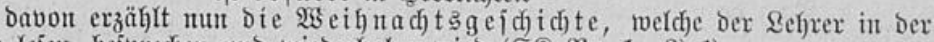

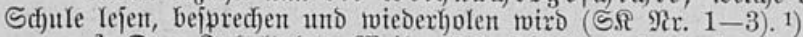

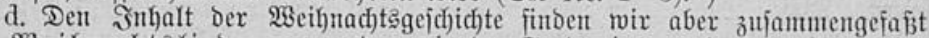

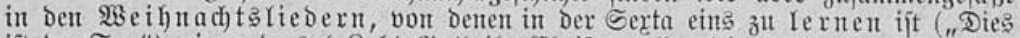

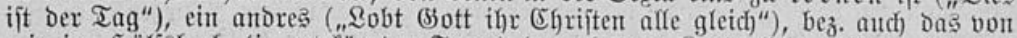
mix in Sülfisbud) (in vertïrzter Jorm) Dargebotene Qutherlied: "Som Simmel hoch" gelejen und natürlidb bejprodben merben mag. ${ }^{2}$ )

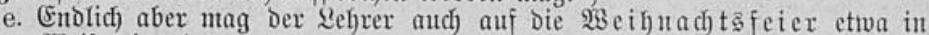
folgender Weije eingehen.

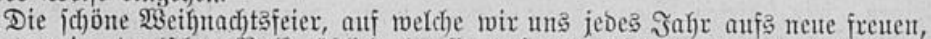
ijt fajt mux im Deutichen Bolfe ïblid), Da fie auf Den Sitten unjerex alten Dor:

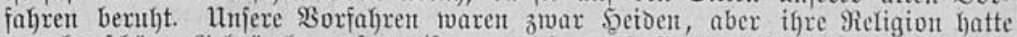

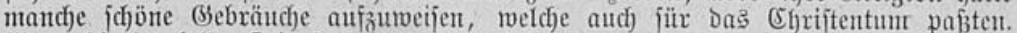
Wem die Dunfelite Beit bes Jahres herbeigefonmen war, wo man hoffen Durite,

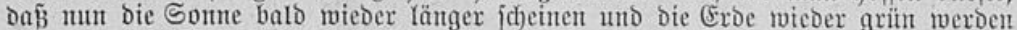
werbe, Dan pflanzten an Dem Jejte, Das jie einem ihrer (Sötter zu Ehren feierten,

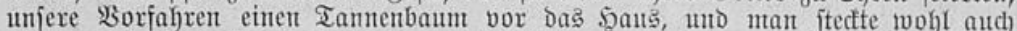

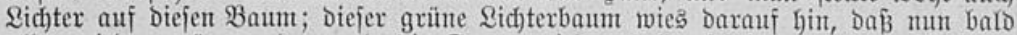

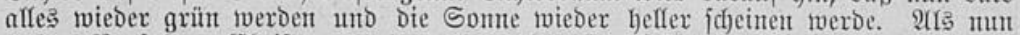
unjere Borfahren Elyriften wurben, Da gaben fie bieje beidnijd)e Sitte nidgt auf, aber jie feiertent mu bas bisher heionijche freit in d)riftlichem Sinne. Bei Dent

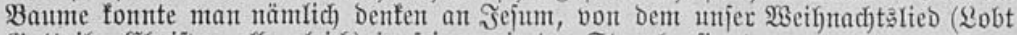
(Sott ihr (efrijten alle gleid)) in jeiner vierten Strophe fingt:

"Dem ex ift Davio's Reis,

Das jeinem Stamm entiprié̃en jollt

Эn Diejer Yebten Beit."

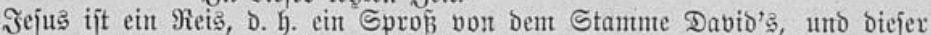

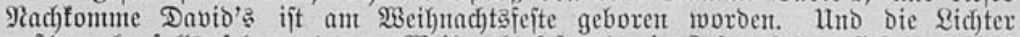

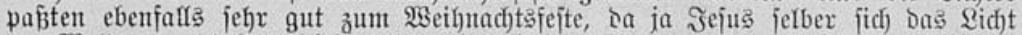
Der Welt genamt hat; Diejen (Sebanfen finden wir ausgejprodhen in Der vierten

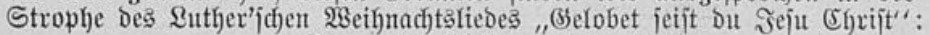

Das ew'ge Qid)t geht Da herein,

(siebt Der Welt ein neuen Schein;

(F. Ieud)t wohl mitten in Der Nad)t

Unto uns Des Sidjtes Rintoer mad)t.

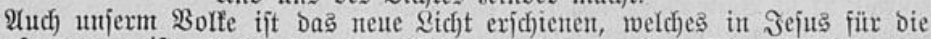

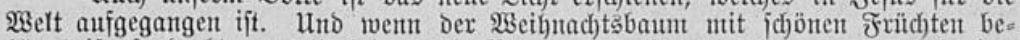
hängt iît, fo Dadjte man Dabei an Die Bäunte Deß 'Barabiejes, uno man bebadjte,

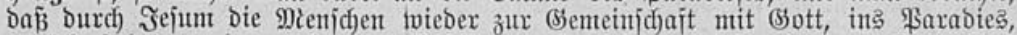

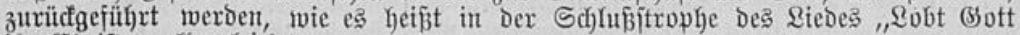
ifgr (Ehriften alle gleidg":

,Seut jod)leuñt ex mieder auf die Thür

Bum jübuen \$arabeis;

Der Eherub iteht nidjt mehr bafür;

(Sott jei \&ob, (E) r' und 'Breis!"

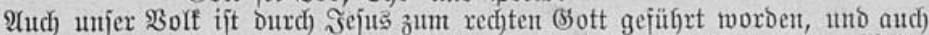

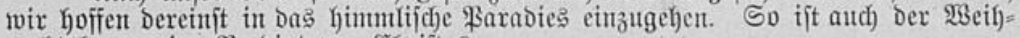
nachtsbaum eine \$redigt von Ehriftus.

f. Went mun Das Beilynad)tsfejt vorïber ijt, bann feiern twir ant 31 . December

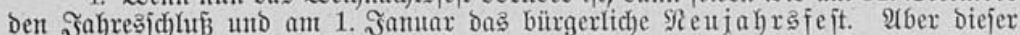
Tag hat aud eine Bebentung für Das Stirdjenjafyr; am adjten Tage nad) Der

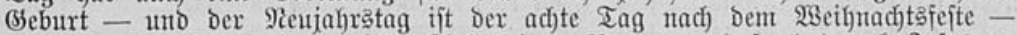

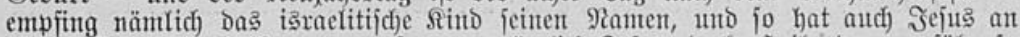

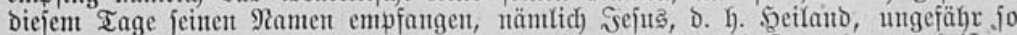

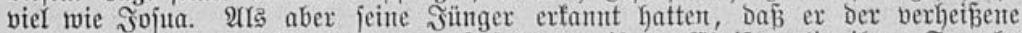

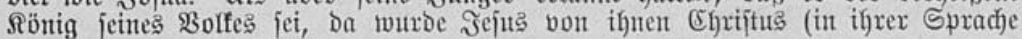

1) Weut ber Sehrer Beit ïbrig hat, faun er biex aud ben Sdjüler etwas von Bethlehem,

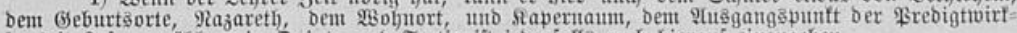

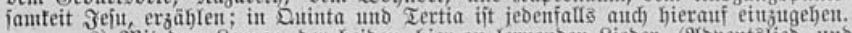

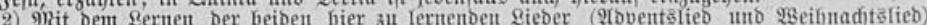
mun ber Qebrer natïrlid) etnvas eber anfangen, ba jonjt bie-3eit ju fuapp wiro. 


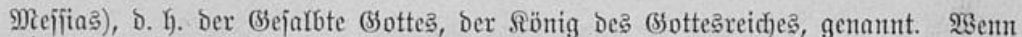

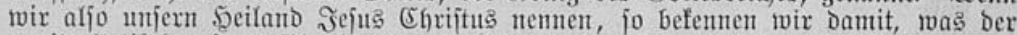

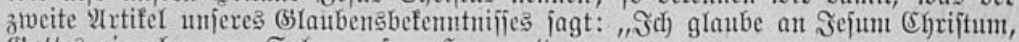
Bottes eingeborenen Soln, umjern Serrn."

g. Rach Dent Reujahrafeite feterten aber bie alten Chrijten (und nod) heute

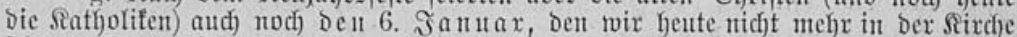

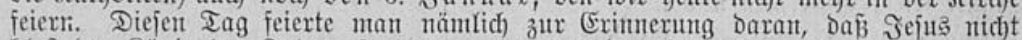

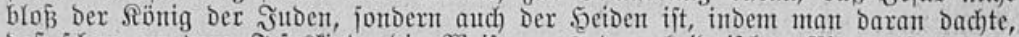

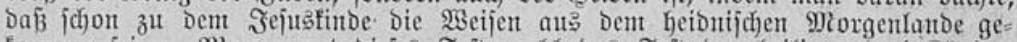
fonmten jeien. Man nemt Diejes Jeit wohl bas Jejt Der heifigen Drei Rönige, weil die fpätere Sage, von weldjer aber nichts in Der Bibel iteht, erzärlte, Dấ

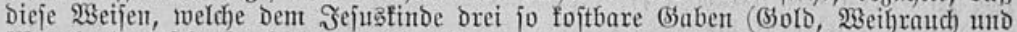
Miyrrben) gej(hentt haben, brei Rönige aus Dem Siorgenlande getwejen jeien.

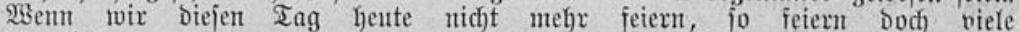
Semeinden an biejem ober an einem andern Tage ein פijifionsfeft, an meldjem

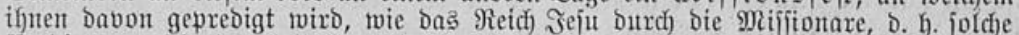

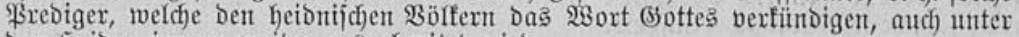
Den Geiben immer meiter ausgebreitet iviro.

Son Den 1450 Mifll. Menjoben, die es nämlidy Geute giebt, finto exit 400 Mrifl. Ehrijten geworden. Da mu Jejub befohlen hat, Daß̉ wir allen Menjchen das Evangelium prebigen follen, jo haben die (Shrijten aud) immer aufg nente \$rebiger

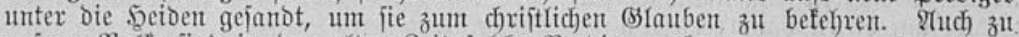

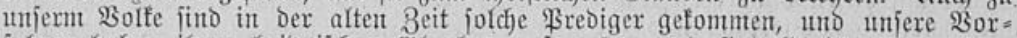
fahren haben ihren heibnifchen (Slauben aufgegeben und fint (Shrijten geworbent.

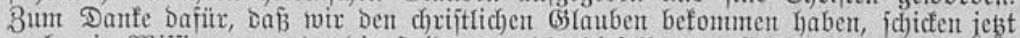
aud) wir Meifjionare unter bie Seiben, weldje Diejelben zlt Ehrijten madjen jollen. Das thut die evangelijaje Rirche etwa jeit humbert Jahren, und es iit diejen. \$rebigern gelungen, in Den verjobiedenten Sänbern etwa jhon' 3 Mill. Seiben zum evangelifdjen (Staubent zu befeljrent. 2tber es twird noch lange Dauern, eke die

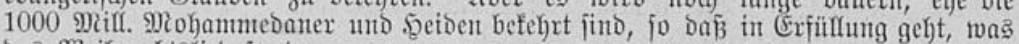
Das Weifuachtslied jagt:

Dies ijt Der Tag, Den (Sott gemad)t,

Sein toerd' in alyer 23 elt gebad)t;

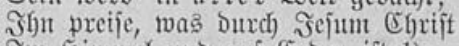

Эัm Simmel und auf Eroen ijt. ${ }^{1}$ )

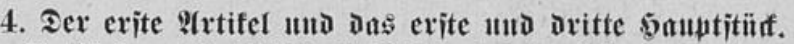

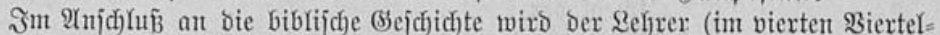

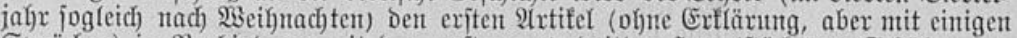
Spriichen) in Berbindung mit Dem eriten und Dritten Sgauptîtücf Dem Schüler, Der

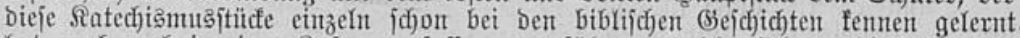
hat, aud) nod) in einer 3ujammenfaffung vorfïloren, welebe fich an bie bibl. (Se=

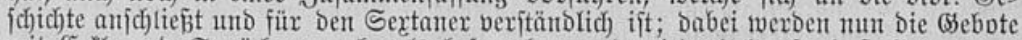

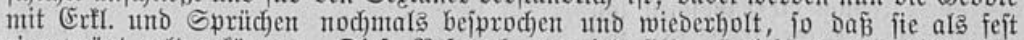
eingeprägt gelten fömnent. Dieje Bejpred)ung wiro fiḑ mögliđjit an Die bibl. (Se=

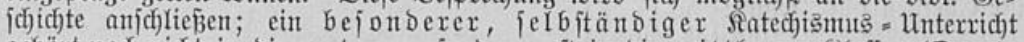
gehört nod) nid)t in bie unteren, jonbern exit it Die mittleren Silafien (Duarta und Tertia), two ex aber um Der in Diejem 2ilter exfolgenten Eonfixmation willen umentbelyrlich) ijit.

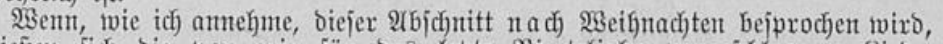

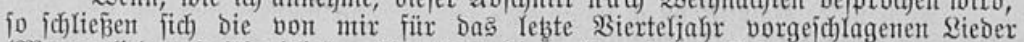

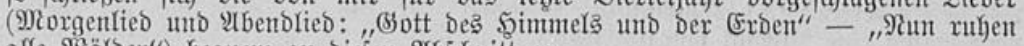

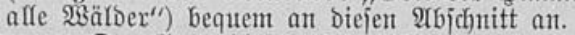

Dex (Santg Diejes Unterridyts fömnte etwa folgender jein. 2)

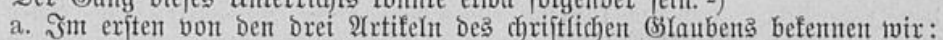
"Iฐ) glaube an (5) ott." Bwar giebt es nod) heute viele Menjichen, Die Sgetben,

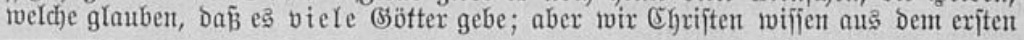

1) Jür Sexta ift wohl fier ber redte Drt, un bent Sđjüler ein Wort über bie Mififiton zu

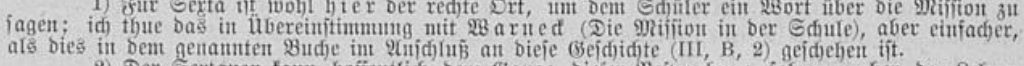

2) Der Sertaner fanm hoffentfid) Dem (Sange Diejer Beipredumg folgen; aber ber Qebrer

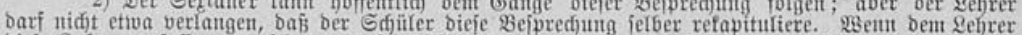

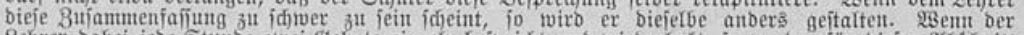
Sehrer babei jebe Stumbe jwei Gebote einjeln beipridit unb twieberholt, jo werben fir biejen शabidutt

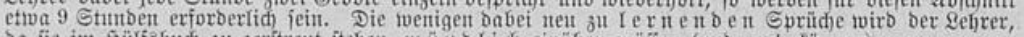

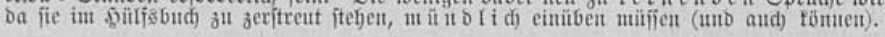




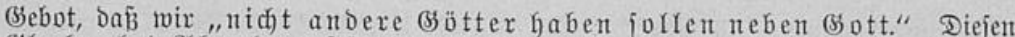

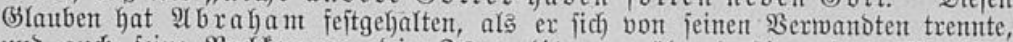

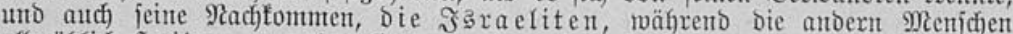
allmähliç şei ben getworben finto.

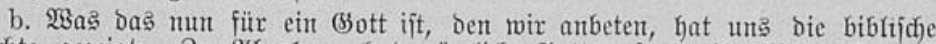
Gejejichte gezeigt. But 2ubraham hat nämliç) (Sott gejagt: (1. Moje 17, 1) 1) "I̧ bin Der allmäctige (Bott, wanble vor mix und jei fromm!" Der

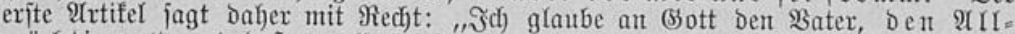

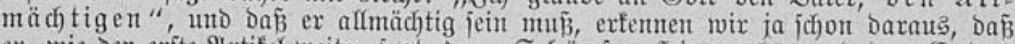

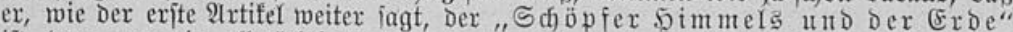
iit, bent mur ein allntächtiger Bott fonnte Shimutel uno (Erbe id)affen. WSer aber

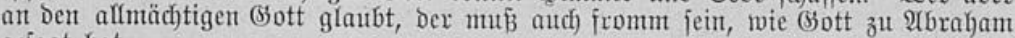
gejagt hat.

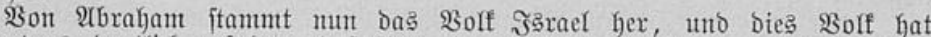

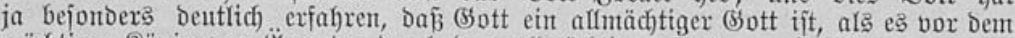

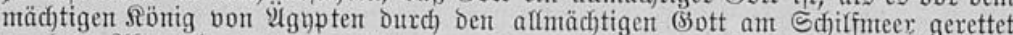
murbe. Aber als es mut gerettet war, Da empfing es von Mojes am Berge Sinai

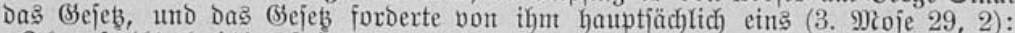

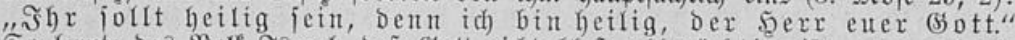

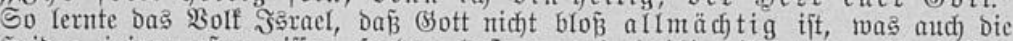

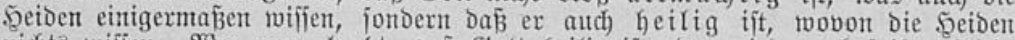
nichts wifien. $2 \mathrm{Ser}$ mun glaubt, $\mathrm{Da} \tilde{\beta}$ (Sott heilig ijt, Der wiro aud) jelber heilig

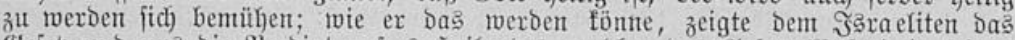

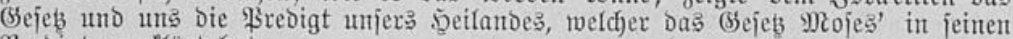
Freoigtent erflärt hat.

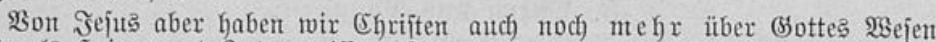

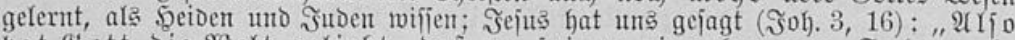

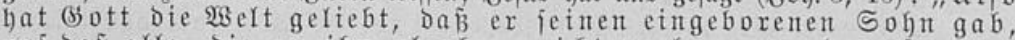

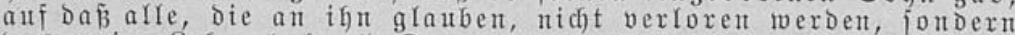

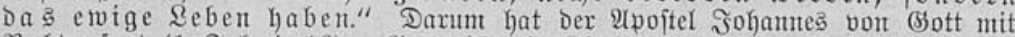

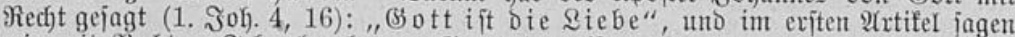

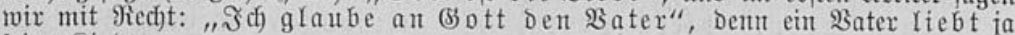
jeine Rimber.

WSen aber (Şott uns liebt, bann werben wir ifn auth twieber lieben, uno

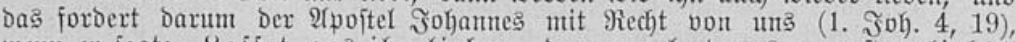
wem ex jagt: "Laffet ung ihulieben, benn ex hat ung zuerit geliebt."

c. TSem wir aber bsott lieben, jo werben wix aud zu ifm beten; wie wir aber betent jollen, hat uns รejuß im Saterunjex gezeigt.

Den allmä d)tigen (Sott bitten wir um Das tägliche Brot (4. Bitte), Daڤ

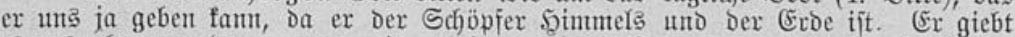
eక uns aber nux bam, went wir arbeiten; wir bürfen es uns nidjt Durch Stehlen ertwerben (Bsebot 7 ).

Den heiligen Bott bitten wir um \$ergebung Der Sïnden (Bitte 5) unt Betvalyrung vor neuer Sïnbe und vor Dem lubel (Bitte 6 u. 7).

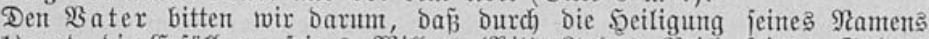
(Bitte 1) uno bie Erfüflung jeines rsitfens (Bitte 3) Das Reid) jeines Sohntes fonme (Bitte 2), weld)es er Durd) Miojes uno Die \$ropheten vorbereitet uno Durd) รejum gegrünbet hat.

Daß wir unt alle bieje Dinge int Ślauben bitten Dürfen uno bittent jollent,

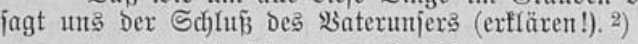

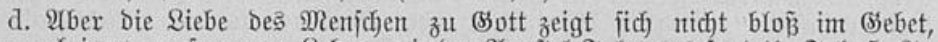

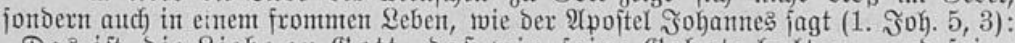
"Das ift Die Riebe zu Bott, bá̉ wix jeine Bebote halten, und feine "Sebote jino nidjt jidjer."

Die Bebote (Sottes jagen uns aber zuterit, was wir Brott felber jujulbig fitio (ङebot 1-4).

Jnt exiten Bebot wiro von uns gefordert, wie \&uther in Der Erffärunt mit Fecht jagt: ",Wir jollen (Sott über alle Dinge (D. h. mehr als alle Dinge) fürchtent,

1) Diejer tvie alfe folgenben nod) nidjt gefernten Sprïdje biejeß 2(bjduttteß merben bei ber Beipred)ung Dem Ğd)îler engeprägt.

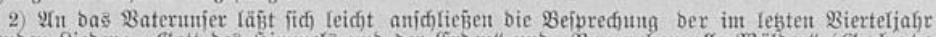

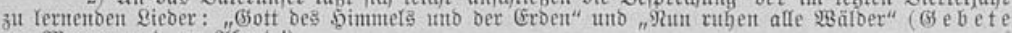
ant Porgen und an 2ै6ent !). 


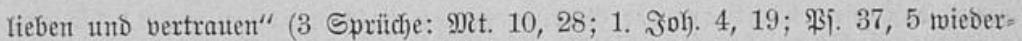
Golen unb beiprecten!)

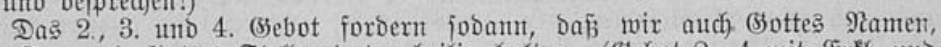
Sottes Tag unt Sottes Stellvertreter heilig halten. (Bebot 2-4 mit Exfl. unt) Spritchen beiprechen uno wiederfolen; von Den Sprïchen bei Schula find früher gelernt worden, bez. jeşt zu lernen: Mit. 12, 36; Mit. 5, 37; \$i. 50, 15. -

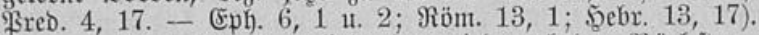

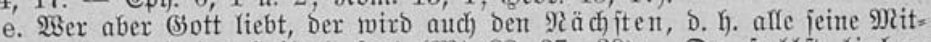
meniffen, lieben, wie ber seiland jagt (Mit. 22, 37-39): "Du jollit fieben (3) ott Deinen Serrn von ganzem $5 e x z e n$, von ganzer Seele und von ganzem Gemitte; Dies i jut Das vornehmite und größte Gebot; Das andere aber ift dem gleid): Du porfit beinen siäditen lieben als bich jerbit."

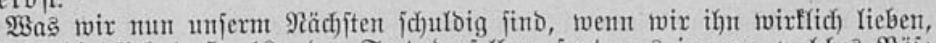
Daß zeigent bie Brebote 5-10; Der Tert Derjerben jagt uns inmter, weldjes Böje

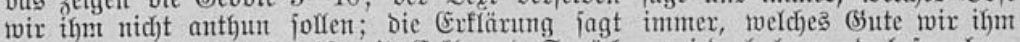
thun follen. (Sebot 5-10 mit Exfl. unt Epriid)en wieberholen uno bejprechen; von ben Spruidjen bei Sidnulz find früher gelernt worben, bez. jebt zil Yernen: 1. Moje 9,6 ; Эaf. $1,19-20 ; 1$. Joh. $4,20-21 ; 1$. Timt. 6,$6 ; 1$. Tim. 6 , 9-10. - Eph. 4, 25).

f. (Sottes Bebote jollen wir aber nidjt blop lernen, jonbern aud halten;

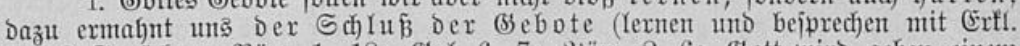

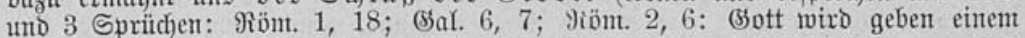
jeglichen nad) jeinten $\mathfrak{B e r f e n t}^{1}$ )

\section{Diteru แni Pingiten; ${ }^{2}$ ) \\ Dex 3 weite utio oritte $\mathbf{9}\left(\mathrm{rtife}^{3}\right)$}

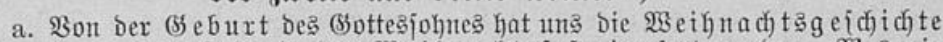

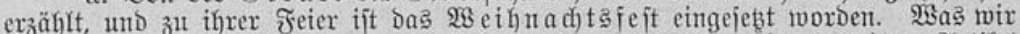

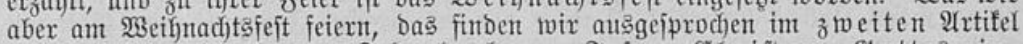

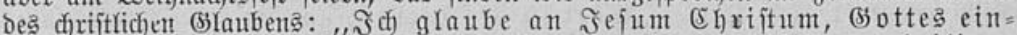
geborenen Sohn, unern Serru, ber empiangen ift vom heiligen Seifte, geboren oon ber Jungfrau Maria."4).

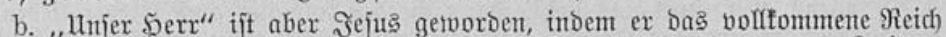
Bottes gegrünbet Kat, zutädjit Durd) jeine \$rebigt: "Na d bem vor Beiten (sott mandhal und manderlei 2 eije gerebet hat zu ben

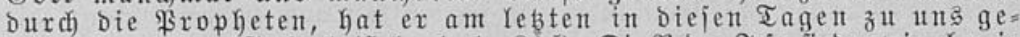

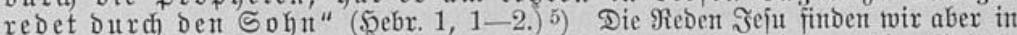
Der Keiligen Sdjrift aufgezeidgnet, uno an vielen Sonntagen wiro ums im Bottes= Dienfte von jeinen Reben etwas vorgelejen und oft auth in ber Frebigt erflärt.

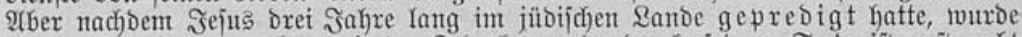

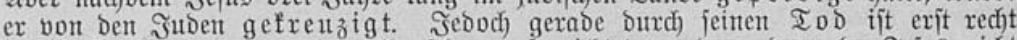

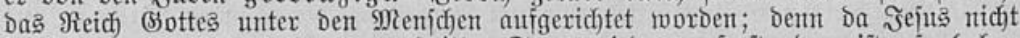
im Tobe geblieben, jondern an Dritten Iage wieber auferftanden ift, io faben

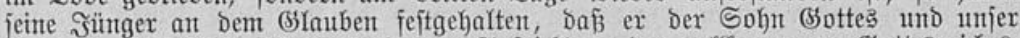

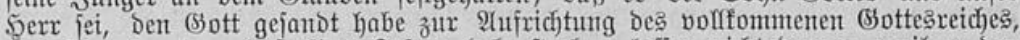

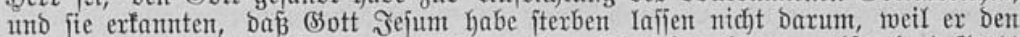
Tod verbient hatte, fondern aus Siebe zu Den Menjiden, Demt "aljo hat Bott

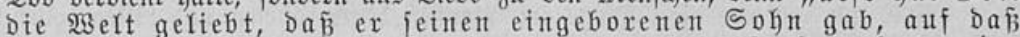
alle, bie an ifn glauben, nidgt verloren werben, jondern das ewige

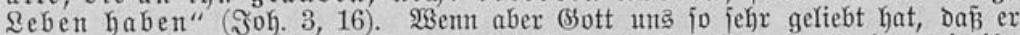
jeinen eingeborenen Sohn für ung in Den Iod gegebent hat, fo werben wir ifhn audf) mieber lieben.

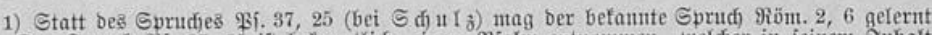

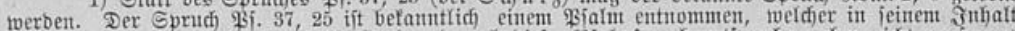

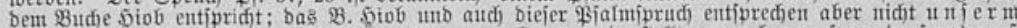

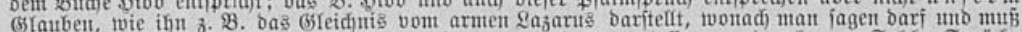

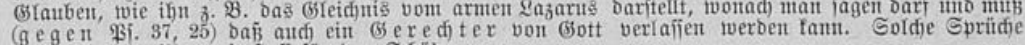
inb aljo tein Miemorieritofi für ben Śdjüler.

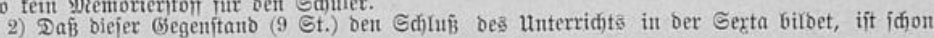
oben bemerft roorben.

3) Die bei biejer Beipred)ung angefüliften Bibeliprïd)e werben in be r St un b e gelernt; ber aweite unb britte 2urtifel werben zutadjt abjdnittweije, twie fie hier bejprodjen werben, in ber Sdjule gelernt uto julest als Ganzes feit eutgeprägt.

4) Der Dext Des Safechismus ift fur zu extlären.

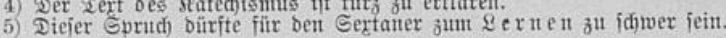




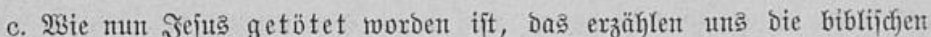
(Sejdidften, meldye wir nad) Dem Reuen Teftamente im zmeiten Teil umjerer "biblijdent (Sejchidbte" finden: ${ }^{1}$ )

ธึ 22-23. Dex Einzug in Jerujalem (ßalmionntag);

ธ尺 26-29a. Dex Iebte Ubend (grïner Domeritag);

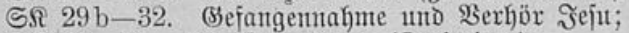

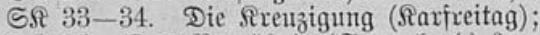

ธึ 35. Das Begräbris (Sonnabend). ${ }^{2}$ )

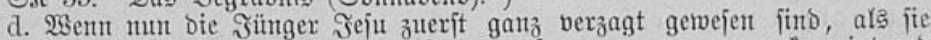
jahen, twie ihr geliebter Meipter von Den Juben gefangen genommen, gefrenzigt uno

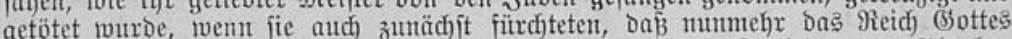

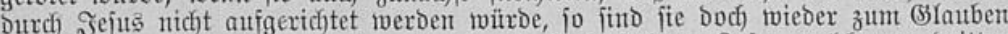

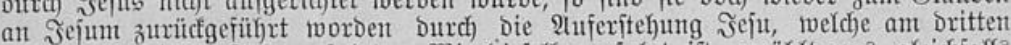
Tage nach jeintent Dode exfolgte. Wie biejelbe exfolgt ift, exzählt uns gleidjalls unjer Bucb:

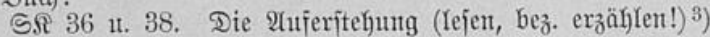

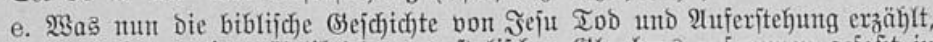

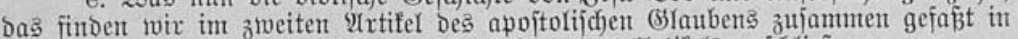

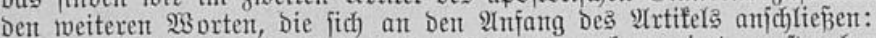

(5)litten unter \$ontio \$ilato, gefreuzigt, gejtorben und be

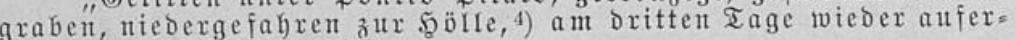
itanden von Den Toten."

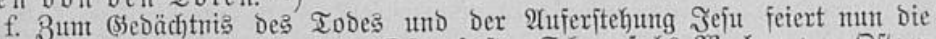

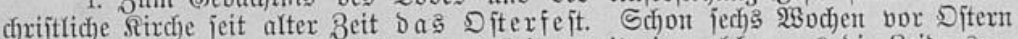

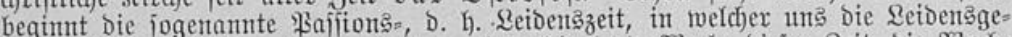

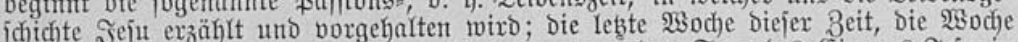

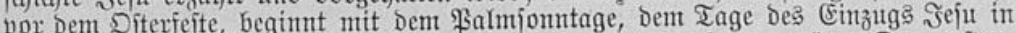

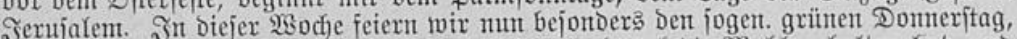
Den Iag, an twelchem Jejus mit jeinen Jüngern Das leb̧te Mahl gehalten hat, und Den Rarfreitag, Den Tobestag Эeju. Derfolgende Somtag, Der Diterjonntag, ift Der

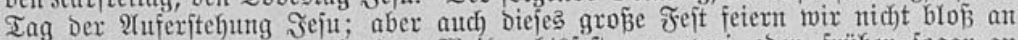
einem Tage, jondern, wie Daక Weifnadjtefeit, an zwei oder früher jogar an brei Tagen.

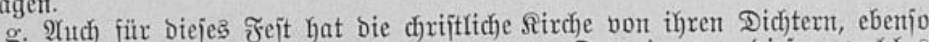

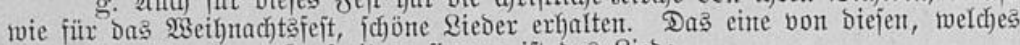
wix in Der Regel an fraxireitage fingen, ijt Das 2ied:

"D Şaupt voll $\mathfrak{B}$ lut und $\mathfrak{W}$ unden;"

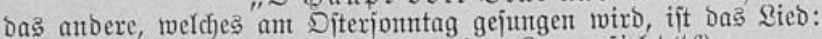

"Эeius meine 3 uveriid)." ${ }^{6}$ )

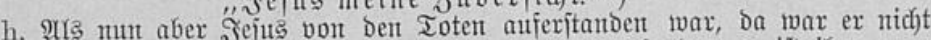
mehx, wie früber, bauento mit jeinen Ssüngern zujammen, jonbern ex ijt ihnen nux

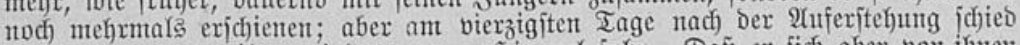

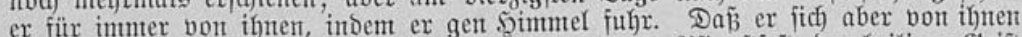

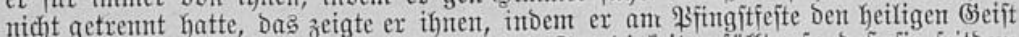

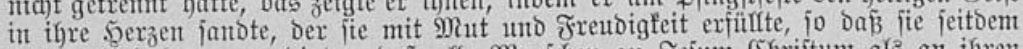

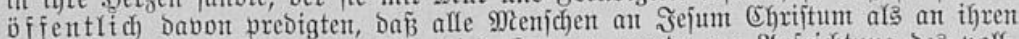
Seilanto glauben jollten, Der einjt wieberfonmen werde zux 2ufridjtung Des voll= fommenen (Sottesreiches.

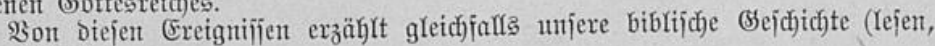
ঢеว. exz̆ählen!):

S̊ 41. Die Şimmelfahrt;

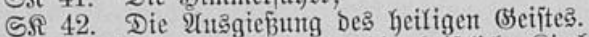

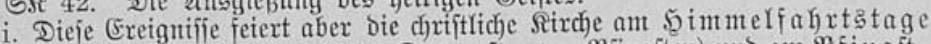
(vierzig Tage nach) Ditern, am vorlebten Donteritag vor \$fingiten) und am \$fing it = feite (50 Tage nad) Ditern), weldyes wix ebenfalls an mehr als einemt Tage feien. $2(m$ Somntage Darauf gebenfen wir bann nod) einmal an alles, was $\mathfrak{B a t e r}$ und Sobn und heifiger (Steift an uns gethan haben uno nod) heute thun - Dấ

1) Der Gehrex tvirb biejelben entweber lejen laijen, ober (went fie aus Mangel an Beit ge= tïrst werben müilien) jelber erzäblen.

2) $\mathfrak{B g l}$. untett g.

3) Bgl. untent g.

5olle - Totenreid, nidit: 2ufenthaltsort ber Berbammten.

5) Dent 2 ortlant nad) einfad) ju erflären! an bie betr. bibl. (Geid)idjten) gelelen unb einfad) erflärt tverben. 


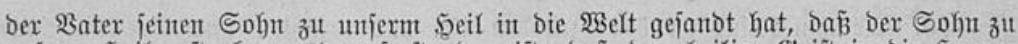

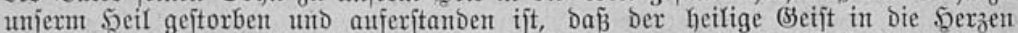

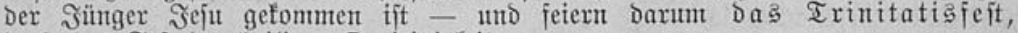
D. h. Daق Feft Der heiligen Dreieinigfeit.

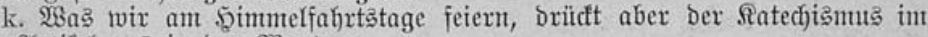
żmeiten 2 rtifel aus in Den 230 orten:

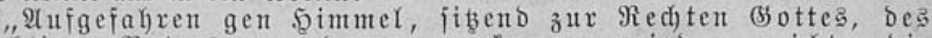
allmäditigen 2 aters, von Dannen ex fommen wirb, zul ridjten bie Sebendigen unto Die Toten. 1 )

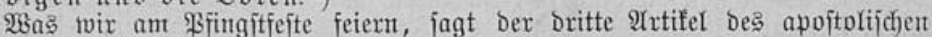
Glaubens mit Den $230 r t e n:$ "If gla glaube an ben heirigen (s) eift."

1. Diejer 2 rtifel weijt aber in Den folgenden Worten aud) nod) Gin auf bas, was ber heilige Seijt an Den Ehriften gethan hat uno nod) hente thut uno Dereinit

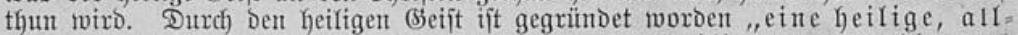
gemeine driftlid)e Rird)e, Die (Semeinde Der Seifigen", zu weldjer aud) wir gehören. Durd) Den heiligen (Seijt wird in uns getwirft Der (Blaube an Die "Dergebung Dex Gïnben", unD ment wir Durch Den Keiligen (Seij̄t gläubig

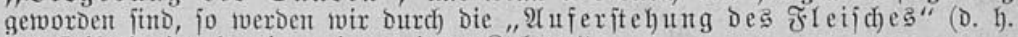

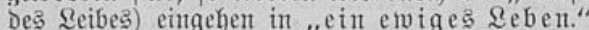

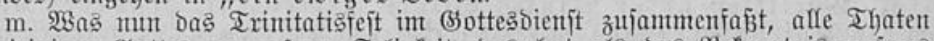
Des Dreieinigen Ssottes zu unjerer Seligfeit, Das hat als Das Befentnis unieres

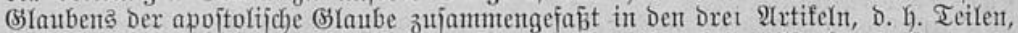
auङ Denen ex bejteht, von Denen Der exite uns hinweijt auf Das 2 serf bes 93 aters, Die Sdjöpfung, Der zweite auf Das Werf Des Sohnes, die Erlöjung, Der Dritte

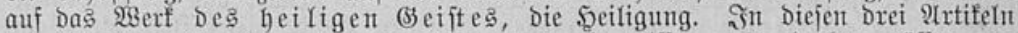

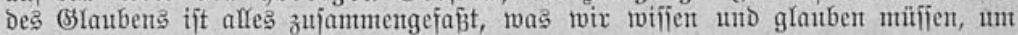
jelig zu werden. ${ }^{2}$ )

n. Damtit mun Das alfes, was פater uno Sohn und Beift für uns gethan haben, uns auf Erben und im .̧immel zu, teil merbe, wollen wix mit bem apoitel Baulus inmer aufs neute beten:

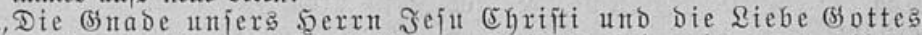
und bie (Semeinjoft bes beiligen Beiftes jei mit uns allen" (2. ก̃or. 13, 13).

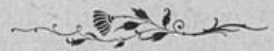

1) Einfađ extlären!

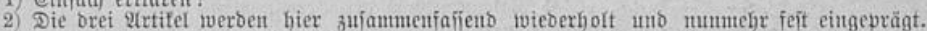

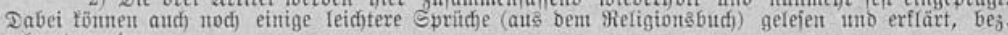
gelernt twerben. 
Der Bater jein unjerm Seil g Der Эünger ? D. h. Das Feit k. $2 \mathrm{as}$ zmeiten 2 rtife „2น $\mathcal{2} \mathrm{g}$ allmächtige Rebendigen พืละ

(Slaubents mit

\section{Dieis}

was ber heilis

thun wiro. I

gemeine d) $x$

wix gehören.

, 2 ergebun?

getvorben fint

Des నeibes) ei m. $\mathfrak{W a}$

Des Dreieiniges

(Staubenta Dex

aut Denten ex

Die Sdjöpfung

auf Das 2 erf

Des (3laubens

jelig zu werde n. Dar

haben, 1แก a1 Raulus intme: "Die ItIt die (2. Ror. 13,

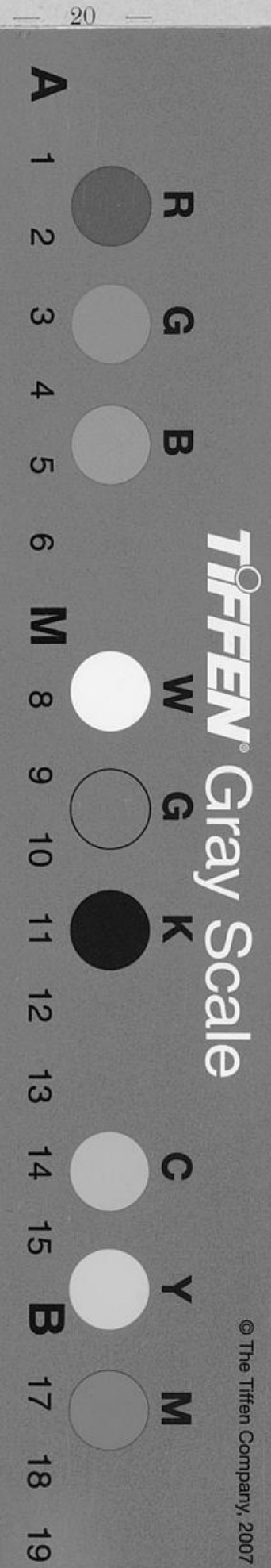

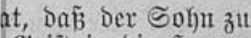
Beijt in Die 5erzen 5 Irintatisfeit, Der Rateçiรnuเ im ten Bottes, Des b, zut riditen bie

fel bes apoitolijdjen

(c) nod) bin auf Das, ife thut uno bereinit eine heirige, all= n", zu meldjex aud $t$ Der Maube an Die eiligen Breijt gläubig \&leijd)es" (D. h).

nenfañt, alle Thaten 3efentnis unieres Urtifeln, o. h. Teilen,

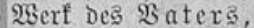
Erlöjung, Der Dritte Diejen orei 2 rtifeln glantben milfien, um

B̉eijt für unङ getban pir nit Dent थpoitel

Die Riebe (Sottes mit uns allen"

munuthr feit eingevrägt. geleíen unb erflät, be 